\title{
Article \\ The Moderating Role of Personality in the Relationship between Internet Use and Study Abroad Difficulties
}

\author{
Gregory-Siy Ching (D)
}

check for updates

Citation: Ching, G.-S. The

Moderating Role of Personality in the Relationship between Internet Use and Study Abroad Difficulties. Int. J. Environ. Res. Public Health 2021, 18,

7707. https://doi.org/

10.3390/ijerph18147707

Academic Editor: Paul B. Tchounwou

Received: 29 June 2021

Accepted: 19 July 2021

Published: 20 July 2021

Publisher's Note: MDPI stays neutral with regard to jurisdictional claims in published maps and institutional affiliations.

Copyright: (c) 2021 by the author. Licensee MDPI, Basel, Switzerland. This article is an open access article distributed under the terms and conditions of the Creative Commons Attribution (CC BY) license (https:// creativecommons.org/licenses/by/ $4.0 /)$.
Graduate Institute of Educational Leadership \& Development, Research and Development Center for Physical Education Health and Information Technology, Fu Jen Catholic University, New Taipei City 24205, Taiwan; 094478@mail.fju.edu.tw

\begin{abstract}
Studying abroad can be stressful due to culture shock and various other difficulties. However, with the current prevalence of information communication technology, we can surmise that study abroad difficulties should be minimal. Since it has been shown that an individual's personality is highly associated with their internet use behaviors, it would be interesting to determine the effects of personality traits on the relationship between internet use motives and perceived study abroad difficulties. Data were collected from 1870 volunteer study abroad students in Taiwan. Hierarchical regression analysis revealed that when controlling for the effects of age, gender, duration of stay, student status (short-term exchange or degree-seeking), and internet use motives (online benefits, habits, and facilitation), the personality trait neuroticism consistently showed significant relationships with the various study abroad difficulties. Moreover, moderation analyses revealed that all the personality traits except conscientiousness showed significant interactions with internet use, while simple slope comparisons showed significant differences between the high personality traits and their lower counterparts. In sum, an examination of the moderating role of personality traits in the relationship between internet use and study abroad difficulties may be useful for preemptively identifying at-risk students.
\end{abstract}

Keywords: adaptability; culture shock; study abroad; higher education; personality

\section{Introduction}

The COVID-19 pandemic has brought much uncertainty with respect to studying abroad [1]. The temporary closure of universities and travel bans across the globe have resulted in disruption to study abroad plans. This has resulted in study abroad programs focusing more on shorter travel distances and particularly East Asia as an emerging regional destination hub [2]. For many, study abroad plans have just been delayed or postponed. Recent surveys have indicated that the desire to study abroad is still strong [3,4], prompting universities to reinvent and prepare themselves to facilitate studying abroad in the postpandemic future [5].

Up until the pandemic, Taiwan was actively involved in promoting international academic exchanges [6]. As a result of the new Southbound Policies [7] and student recruitment in Mainland China [8], in 2019, there were around 130,000 study abroad students in Taiwan [9]. According to the Ministry of Education [10], the majority of the study abroad students in Taiwan are from within the regions of Mainland China, Malaysia, Hong Kong, Japan, Macau, Vietnam, South Korea, Indonesia, Thailand, and India, which are the top ten contributors, making up almost $80 \%$ of the international student enrolment. Taiwan is also ranked by Quacquarelli Symonds (QS) as the second best place to study in Asia [11]. In addition, Taiwan also ranked 19th in the QS Higher Education System Strength Rankings with 43 universities entering the latest Asian rankings (five in the top 50 with National Taiwan University in 19th place) [12]. With this having been said, Taiwan study abroad students can be considered as a point of interest. 
Studying abroad is not without its challenges [13]. It has been shown to be a stressful undertaking [14-16] that can affect an individual's mental well-being $[17,18]$. Study abroad students may experience culture shock resulting from their encounters with an unfamiliar culture [19]. Importantly, culture shock is not limited to outgoing students but is also common among returning (or re-entry) students [20,21]. Nonetheless, many still consider that the positive experiences gained from studying abroad are sustainable [22] and outweigh the perceived negative aspects [23]. Hence, it is still considered rewarding for students to participate in study abroad opportunities.

With advances in information communication technology, the internet provides academic sojourners the opportunity for instantaneous communication [24,25] and the facility to document their experiences [26], while also reducing depression levels [27]. Furthermore, studies have found that the internet can provide access to a wide social support network, which can help foster confidence in those studying abroad [28]. At the same time, these familiar co-national social networks provide information and emotional support for individuals in unfamiliar environments [29]. However, too much dependence on these co-national networks may hinder their cultural learning goals [30-32]. In general, however, the internet enables students to feel more connected with their home (family and friends), while also facilitating and enhancing their study abroad experiences [33].

Research on studying abroad has found that students' personality traits are related to their intention and decision to participate in study abroad programs [34,35]. Importantly, intercultural competency development is also related to certain personality traits [36,37]. For instance, intercultural effectiveness is said to be positively correlated with extroversion and openness [38], and conscientious students tend to be more cautious in choosing between study abroad programs [39]. In most of these studies, the five factor model [40] or the big five personality traits [41] are typically used [42]. The personality traits openness, conscientiousness, extraversion, agreeableness, and neuroticism represent the various stable individual differences within the thoughts that people have, the feelings that they experience, and their behaviors [43]. Within study abroad studies, these five personalities are commonly used to understand and describe how students are able to adjust to their new experiences. Research findings have shown that students' openness is directly associated with their tendency for diversity, which in turn led to better adjustment [31,44], while high levels of agreeableness and openness predict the desire to study abroad [42].

Importantly, personality traits also play an important role as a moderator for technology use [45]. For instance, a study on German and Chinese individuals showed that higher levels of neuroticism and lower levels of conscientiousness are strongly associated with problematic internet use [46]. Furthermore, students' attitude toward social media was moderated by their degree of openness and neuroticism [47]. Given these findings and those of other studies, which suggest that an individual's internet use habits are highly related to their personality [48-50] and with the environment with which they interact [51], it is therefore interesting to determine whether study abroad difficulties are affected by an individual's internet use and personality.

Although the relationship between personality traits in study abroad students and internet use has been studied, evidence of the moderator effect of personality traits (openness, conscientiousness, extraversion, agreeableness, and neuroticism) on the relationship between internet use motives and study abroad difficulties is limited. Thus, the objectives of this research are as follows:

- To determine the role of personality traits in predicting study abroad difficulties;

- To determine the moderator effect of personality traits on the relationship between internet use and study abroad difficulties;

- To determine the differences between high and low personality traits with regard to the relationship between internet use and study abroad difficulties.

Age, gender, duration of stay, and status (short-term exchange or degree-seeking) of study abroad students in Taiwan (see Figure 1 for the conceptual diagram of the moderation model) were controlled in the analyses. 


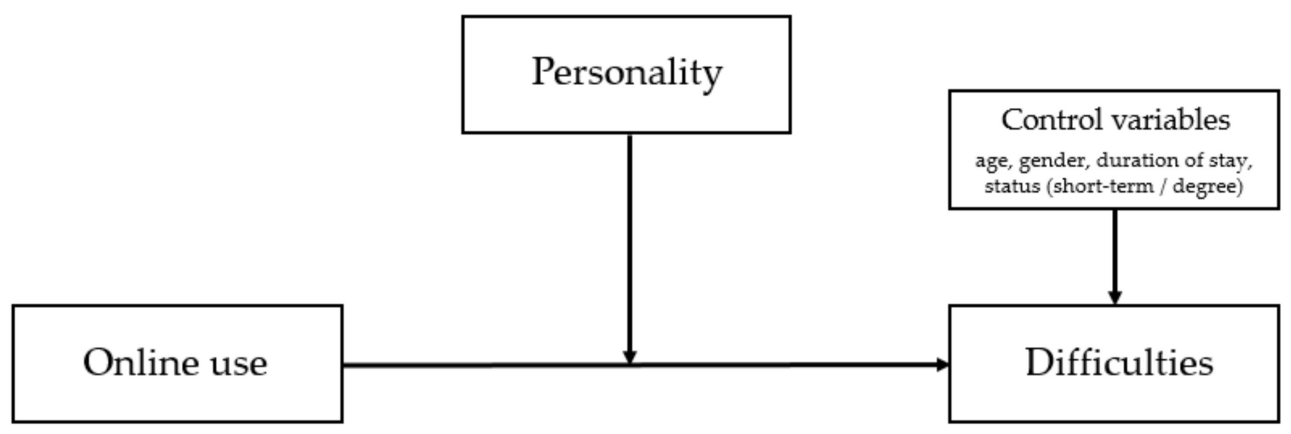

Figure 1. Conceptual diagram of the moderation model.

\section{Materials and Methods}

\subsection{Study Design and Participants}

The current study is designed as a quantitative study, whereby data are collected using an online survey and later generalized across a group of people to explain a particular phenomenon $[52,53]$. Posters advertising the study were mailed to international student offices in universities throughout Taiwan. As an incentive, a convenience store cash coupon was offered to the first 500 respondents. A brief description of the study and an explanation of how the collected data would be analyzed and used were provided together with the consent form. Furthermore, participants were informed that the survey included not only personality questions but also their everyday experiences in Taiwan. The study protocol was evaluated and approved by the Fu Jen Catholic University Institutional Review Board.

Data collection and analyses were completed by means of an online survey using the volunteer sampling technique that took place over one 18-week semester during the 2015 academic school year [54]. Sampsize program [55] was used to calculate the minimum sample size. Since there are approximately 112,000 study abroad students in Taiwan during that academic year, a minimum sample size of 383 students was needed for this study (with a 5\% margin of error and 95\% confidence level). The inclusion criteria included students whose nationality is not Taiwanese and who were enrolled in a university either on a short-term exchange (including Mandarin Chinese language center students) or a degree-seeking program. Foreign students enrolled in senior high schools or lower were excluded in this study.

A total of 1958 volunteer study abroad students in Taiwan participated in the data collection. Of these, 88 students withdrew from the study after reading the informed consent form. Information collected from the remaining 1870 participants were analyzed and screened for outliers, and missing data, which accounted for less than $10 \%$ of the entire dataset, were imputed using the expectation maximization algorithm [56,57]. Cronbach's [58] alpha reliability of the entire survey was computed as 0.84 , denoting acceptable internal consistency [59].

Table 1 shows the demographic profile of the students, including the number of female and male participants (female $=925$ or $49 \%$, male $=945$ or $51 \%$ ). The average age of participants was around 26 years old. The status, or study abroad type, of the students is also shown in Table 1. Short-term exchange students accounted for $980(52 \%)$ of the participants, while the remaining 890 (48\%) were long-term degree-seeking students. Shortterm exchange students are typical academic sojourners who are on language programs, cultural immersion stays, and/or academic programs with partner institutions. The typical duration of stay for these exchange students ranges from a few months to a semester and up to a maximum of one year. Degree-seeking students are those formally enrolled in undergraduate or graduate courses with the intention of earning a diploma. The average duration of stay for all the participants was around 15 months. 
Table 1. Demographic profile of the participants.

\begin{tabular}{cccc}
\hline Demographics & Classification & $\boldsymbol{n}$ & $\boldsymbol{\%}$ \\
\hline \multirow{3}{*}{ Gender } & Female & 925 & 49 \\
& Male & 945 & 51 \\
& Total & 1870 & 100 \\
& Short-term exchange & 980 & 52 \\
Status & Degree-seeking & 890 & 48 \\
& Total & 1870 & 100 \\
\hline
\end{tabular}

Note. $n=1870$.

\subsection{Measures}

For background demographics, participants were asked to provide their age, gender, duration of stay, and status (short-term exchange or degree-seeking). Personality traits were assessed using the 44-item Big Five Inventory (BFI) developed by John and Srivastava [60], which collects self-reported agreement on personal behaviors using a five-point Likerttype [61] scale, with ratings from 1 (least agree) to 5 (most agree). The BFI is a commonly used scale for assessing an individual's levels of openness, conscientiousness, extraversion, agreeableness, and neuroticism. Openness or openness to experience is a dimension of personality that describes individual differences in seeking, detecting, comprehending, using, and appreciating complex patterns of information, whether sensory or abstract [62] Conscientiousness can be considered as a tendency to follow socially prescribed norms, to have goals, to plan, and to be self-disciplined [63]. On the other hand, extraversion is characterized by an individual's ability to successfully engage in various aspects of their lives, and they are generally seen as happy, enthusiastic, confident, energetic, and actively involved throughout their lives $[64,65]$. Furthermore, agreeableness is an individual difference that refers to the tendency to be likeable, pleasant, and harmonious with others [66]. Finally, neuroticism describes someone who reacts poorly to environmental stress, who interprets ordinary situations as threatening, and who experiences minor frustrations as hopelessly overwhelming [67]. Cronbach's alpha reliability of the BFI was computed at $0.68,0.72,0.68,0.65$, and 0.69 , respectively, which indicates that internal consistencies of the BFI were satisfactory.

Internet use motives were assessed using the Study Abroad Internet Use Motives Survey (IUM) developed by Lin and Ching [27] for Taiwan study abroad students, which gathers self-reported agreement on online behaviors using a five-point Likert-type scale, with ratings from 1 (least agree) to 5 (most agree). The IUM is composed of three distinct groups of internet use motives: online benefits, online habits, and online facilitation. Online benefits refer to the notion that the internet is able to alleviate both social and academic difficulties. Online habits or social networking habits refer to how students use social networking sites. Lastly, online facilitation pertains to how students use the internet for social and cultural purposes (p. 1208) [27]. Sample items are "help reduce my academic problems," "regularly interact with my friends through social media," and "look for a cultural event that I will attend." Cronbach's alpha reliability of the IUM was computed at $0.83,0.82$, and 0.78 , respectively, denoting good internal consistencies. For the current sample, confirmatory factor analysis was conducted to verify the factor structure of the observed variables $[68,69]$. In order to assess the validity of the observed variables, several goodness-of-fit criteria were used. Results show an adequate fit with a chi-squared value of 417.12 at $p<0.001$ and degrees of freedom $(d f)=41$, root mean square error of approximation (RMSEA) $=0.070$ with $90 \%$ confidence intervals (CIs) of 0.064 and 0.076 , standardized root mean square residual $(\mathrm{SRMR})=0.042$, goodness of fit $(\mathrm{GFI})=0.90$, Tucker-Lewis index $(\mathrm{TLI})=0.94$, and comparative fit index $(\mathrm{CFI})=0.96$, all of which are within the recommended ranges [70-73].

Lastly, study abroad difficulties were examined using the Short-term Study Abroad Situational Change Survey (SSCS) developed by Ching et al. [74] for Taiwan study abroad students, which assesses various self-reported behavioral, cognitive, and affective situ- 
ational change difficulties using a five-point Likert-type scale with ratings from 1 (least agree) to 5 (most agree). Higher mean scores signify higher study abroad difficulties. The SSCS assesses six distinct groups of study abroad difficulties: academic, leisure living, local viewpoints, daily living, responsive, and suppressive. Academic difficulties consist of the cognitive and behavioral changes which occur within the school environment, while leisure living includes a sense of fun and enjoying oneself, with a focus on getting to know more about Taiwan culture. On the other hand, local viewpoints refer to cognitive interpretations of context that focus on local Taiwanese perspectives. As for daily living, it relates to the changes in general living conditions during study abroad. Responsive difficulties are the students' need in overcoming difficulties in dealing with odd situations. Lastly, the suppressive factor refers to the usual situations that students are used to in their home country, but which are difficult to replicate in Taiwan (pp. 60-61) [74]. Sample items are "reading and understanding lesson materials," "going to coffee shops, groceries, or restaurants," "taking a local perspective on cultural issues," "adapting to student life in Taiwan," "dealing with unsatisfactory service," and "being able to use the things that I'm accustomed to." Cronbach alpha reliability of the SSCS was computed at $0.86,0.81$, $0.85,0.81,0.71$, and 0.67 , respectively, denoting satisfactory to good internal consistencies. Confirmatory factor analysis was also performed on SSCS, signifying good model fit with a chi-squared value of 1412.30 at $p<0.001$ and $d f=194$, RMSEA $=0.058$ with $90 \%$ CIs of 0.055 and $0.061, \mathrm{SRMR}=0.048, \mathrm{GFI}=0.93, \mathrm{TLI}=0.92$, and $\mathrm{CFI}=0.93$.

\subsection{Statistical Analyses}

Data were analyzed using SPSS and AMOS (Version 26.0, IBM Corporation, Armonk, NY, USA) on lease agreement from Hearne Software and the freeware Interaction! Software by Daniel Soper (https:/ / www.danielsoper.com/Interaction/, accessed on 5 January 2021). Confirmatory factor analysis, composite reliability, convergent validity (or the average variance extracted), and discriminant validity to validate the IUM and the SSCS were performed using AMOS. For the confirmatory factor analysis, several criteria were used to evaluate model fit: a significant chi-squared value, RMSEA $<0.08$, SRMR $<0.06$, and GFI, TLI, and CFI $>0.90$ indicate a good fit [70-73]. Descriptive statistics, such as mean and standard deviation (SD), correlations among the variables, and internal consistencies of BFI, IUM, and SSCS were all computed using SPSS. Independent samples $t$-tests were also performed to determine whether the students' gender and status (short-term exchange or degree-seeking) had significant effects on their internet use (online benefits, habits, and facilitation), study abroad difficulties (academic, leisure living, local viewpoints, daily living, responsive, and suppressive), and personality (openness, conscientious, extraversion, agreeableness, and neuroticism). Hierarchical multiple regression analyses were then conducted to test for significant relationships between internet use and study abroad difficulties and its subscales while controlling for the background demographic variables age, gender, duration of stay, and status. Lastly, the moderating effect of the different personality traits on the relationship between internet use and study abroad difficulties and a simple slopes comparison between high $(+2 \mathrm{SD})$ and low $(-2 \mathrm{SD})$ personality traits were performed using Interaction! Software [75].

\section{Results}

\subsection{Descriptive Statistics and Correlations among the Variables}

The descriptive results and correlations among the variables are shown in Table 2. The results show that the mean scores of the study abroad difficulties subscales (SSCS factors) ranged from 1.88 to 2.69 , signifying moderately low perceived difficulties. The mean scores of the internet use subscales (IUM factors) ranged from 3.51 to 3.81, signifying moderately high perceived agreement. Composite reliability (CR) and convergent validity (or the average variance extracted, AVE) for the SSCS and IUM factors were all above the cutoff points ( 0.60 for $\mathrm{CR}$ and 0.40 for AVE) and are shown in Table 2 [76]. In addition, discriminant validity (DV) was assessed by comparing the square root of AVE with the correlations of 
the variables. The results show that the DVs were higher than the correlations, signifying adequate construct validity of the SSCS and the IUM [76].

The correlation results show that the study abroad difficulties subscales were positively correlated with each other. Likewise, the internet use subscales were also positively correlated with each other. Interestingly, the study abroad difficulties subscales were mostly negatively correlated with the internet use subscales, implying that as internet use increases, study abroad difficulties decrease. The personality traits openness, conscientiousness, extraversion, and agreeableness were positively correlated with each other but negatively correlated with neuroticism. In addition, openness, conscientiousness, extraversion, and agreeableness were positively correlated with the internet use subscales and negatively correlated with the study abroad difficulties subscales. Neuroticism was negatively correlated with the internet use subscales and positively correlated with the study abroad difficulties subscales, implying that neuroticism is positively linked with study abroad difficulties.

Lastly, duration of stay was negatively correlated with leisure living difficulties, with $r(1870)=-0.07, p<0.01$, and local viewpoints difficulties, with $r(1870)=-0.05$, $p<0.05$. Likewise, duration of stay was negatively correlated with online benefits, with $r(1870)=-0.10, p<0.01$, and online habits, with $r(1870)=-0.05, p<0.05$. This is interesting because it denotes that students who spent less time studying in Taiwan had higher perceived online benefits and habits. In addition, age was positively correlated with daily living difficulties, with $r(1870)=0.11, p<0.01$, and suppressive difficulties, with $r(1870)=0.11, p<0.01$. Surprisingly, age was negatively correlated with all the internet use subscales: online benefits, with $r(1870)=-0.07, p<0.01$; online habits, with $r(1870)=-0.13, p<0.01$; and online facilitation, with $r(1870)=-0.16, p<0.01$. This signifies that older students tend to be less adept at internet usage.

\subsection{Effects of Gender and Status on Internet Use, Study Abroad Difficulties, and Personality}

Independent samples $t$-tests were performed to test whether the students' gender and status (short-term exchange or degree-seeking) had significant effects on their internet use (online benefits, habits, and facilitation), study abroad difficulties (academic, leisure living, local viewpoints, daily living, responsive, and suppressive), and personality traits (openness, conscientiousness, extraversion, agreeableness, and neuroticism).

The results show that statistically significant differences were found for: suppressive difficulties between females $(\mathrm{M}=1.89, \mathrm{SD}=0.73)$ and males $(\mathrm{M}=1.97, \mathrm{SD}=0.84)$, with $t(1844)=2.23, p<0.05$; online facilitation between females $(\mathrm{M}=3.90, \mathrm{SD}=0.79)$ and males $(\mathrm{M}=3.72$, $\mathrm{SD}=0.86)$, with $t(1860)=4.83, p<0.001$; conscientiousness between females $(\mathrm{M}=3.16, \mathrm{SD}=0.62)$ and males $(\mathrm{M}=3.24, \mathrm{SD}=0.62)$, with $t(1868)=2.75, p<0.01$; and neuroticism between females $(\mathrm{M}=2.87, \mathrm{SD}=0.66)$ and males $(\mathrm{M}=2.80, \mathrm{SD}=0.66)$, with $t(1868)=2.52, p<0.05$. Effect sizes were small, ranging from 0.10 to 0.22 [77].

With regard to student status, the results show that statistically significant differences were found for: academic difficulties between short-term exchange $(\mathrm{M}=2.19$, $\mathrm{SD}=0.78)$ and degree-seeking students $(\mathrm{M}=2.29, \mathrm{SD}=0.90)$, with $t(1767)=2.23, p<0.05$; daily living difficulties between short-term exchange $(\mathrm{M}=2.12, \mathrm{SD}=0.90)$ and degreeseeking students $(\mathrm{M}=2.20, \mathrm{SD}=0.95)$, with $t(1825)=2.01, p<0.05$; online facilitation between short-term exchange $(M=3.76, S D=0.81)$ and degree-seeking students $(M=3.87$, $\mathrm{SD}=0.85)$, with $t(1868)=2.85, p<0.01$; openness between short-term exchange $(\mathrm{M}=3.31$, $\mathrm{SD}=0.58)$ and degree-seeking students $(\mathrm{M}=3.37, \mathrm{SD}=0.58)$, with $t(1868)=2.14$, $p<0.05$; conscientiousness between short-term exchange $(\mathrm{M}=3.15, \mathrm{SD}=0.61)$ and degreeseeking students $(\mathrm{M}=3.25, \mathrm{SD}=0.63)$, with $t(1868)=3.59, p<0.001$; agreeableness between short-term exchange $(\mathrm{M}=3.52, \mathrm{SD}=0.56)$ and degree-seeking students $(\mathrm{M}=3.63$, $\mathrm{SD}=0.57)$, with $t(1868)=4.28, p<0.001$; and neuroticism between short-term exchange $(\mathrm{M}=2.90, \mathrm{SD}=0.65)$ and degree-seeking students $(\mathrm{M}=2.77, \mathrm{SD}=0.66)$, with $t(1868)=4.17$, $p<0.001$. Effect sizes were small, ranging from 0.09 to 0.20 . 
Table 2. Descriptive statistics, discriminant validity, and correlation matrix of the variables.

\begin{tabular}{|c|c|c|c|c|c|c|c|c|c|c|c|c|c|c|c|c|c|c|c|c|c|c|}
\hline Variables & PS & Mean & SD & CR & AVE & DV $^{1}$ & 1 & 2 & 3 & 4 & 5 & 6 & 7 & 8 & 9 & 10 & 11 & 12 & 13 & 14 & 15 & 16 \\
\hline 1. Academic & $1 \sim 5$ & 2.24 & 0.84 & 0.86 & 0.51 & 0.71 & 0.86 & 0.48 ** & 0.40 ** & $0.39 * *$ & $0.44^{* *}$ & $0.40^{* *}$ & -0.11 & -0.12 & -0.19 & -0.14 & -0.22 & -0.20 & -0.16 & 0.23 ** & 0.03 & 0.04 \\
\hline 2. Leisure living & $1 \sim 5$ & 1.88 & 0.80 & 0.82 & 0.53 & 0.73 & & 0.81 & $0.33 * *$ & $0.40 * *$ & $0.40^{* *}$ & $0.51 * *$ & -0.08 & -0.12 & -0.20 & $-\underset{* * 11}{0.11}$ & -0.11 & -0.13 & -0.19 & $0.21^{* *}$ & -0.07 & 0.04 \\
\hline 3. Local viewpoints & $1 \sim 5$ & 2.69 & 0.96 & 0.85 & 0.66 & 0.82 & & & 0.85 & $0.31^{* *}$ & 0.35 ** & $0.25^{* *}$ & -0.12 & -0.07 & -0.08 & -0.09 & -0.15 & -0.11 & -0.09 & $0.17^{* *}$ & -0.05 & 0.04 \\
\hline 4. Daily living & $1 \sim 5$ & 2.16 & 0.92 & 0.82 & 0.61 & 0.78 & & & & 0.81 & $0.45^{* *}$ & $0.50 * *$ & -0.05 & $-\underset{* *}{0.06}$ & -0.11 & -0.04 & -0.05 & -0.11 & -0.23 & $0.18^{* *}$ & -0.00 & $0.11 * *$ \\
\hline 5. Responsive & $1 \sim 5$ & 2.47 & 0.92 & 0.72 & 0.46 & 0.68 & & & & & 0.71 & $0.37 * *$ & -0.05 & -0.09 & -0.11 & -0.07 & $-\underset{* *}{0.13}$ & -0.18 & -0.20 & $0.22 * *$ & -0.02 & 0.03 \\
\hline 6. Suppressive & $1 \sim 5$ & 1.93 & 0.79 & 0.68 & 0.42 & 0.65 & & & & & & 0.67 & -0.06 & -0.11 & -0.17 & -0.06 & $-\underset{* *}{0.08}$ & -0.07 & -0.16 & $0.14^{* *}$ & 0.01 & $0.11^{* *}$ \\
\hline 7. Online benefits & $1 \sim 5$ & 3.52 & 0.93 & 0.84 & 0.56 & 0.75 & & & & & & & 0.83 & $0.50^{* *}$ & $0.42 * *$ & $0.13^{* *}$ & $0.11^{* *}$ & $0.10^{* *}$ & $0.08^{* *}$ & -0.07 & -0.10 & $-0.07^{* *}$ \\
\hline 8. Online habits & $1 \sim 5$ & 3.51 & 0.94 & 0.82 & 0.54 & 0.73 & & & & & & & & 0.82 & $0.45 *$ & $0.21 * *$ & 0.20 ** & $0.23^{* *}$ & $0.24^{\text {** }}$ & -0.19 & $-\underset{*}{0.05}$ & $-0.13 * *$ \\
\hline 9. Online facilitation & $1 \sim 5$ & 3.81 & 0.83 & 0.79 & 0.57 & 0.75 & & & & & & & & & 0.78 & $0.22 * *$ & $0.15^{* *}$ & $0.23^{* *}$ & $0.24^{* *}$ & -0.20 & 0.02 & $-0.16^{* *}$ \\
\hline 10. Openness & $1.5 \sim 5$ & 3.34 & 0.58 & & & & & & & & & & & & & 0.68 & $0.29^{* *}$ & $0.28^{* *}$ & $0.24^{* *}$ & -0.21 & 0.03 & -0.01 \\
\hline 11. Conscientiousness & $1.33 \sim 5$ & 3.20 & 0.62 & & & & & & & & & & & & & & 0.72 & $0.31^{* *}$ & $0.35^{* *}$ & -0.45 & 0.01 & $-0.07^{* *}$ \\
\hline 12. Extraversion & $1.25 \sim 5$ & 3.21 & 0.62 & & & & & & & & & & & & & & & 0.68 & $0.27^{* *}$ & -0.42 & -0.01 & -0.02 \\
\hline 13. Agreeableness & $1.56 \sim 5$ & 3.57 & 0.56 & & & & & & & & & & & & & & & & 0.65 & -0.43 & 0.03 & $-0.06^{* *}$ \\
\hline $\begin{array}{l}\text { 14. Neuroticism } \\
\text { 15. Duration } \\
\text { 16. Age }\end{array}$ & $\begin{array}{l}1 \sim 5 \\
1 \sim 312 \\
17 \sim 57\end{array}$ & $\begin{array}{l}2.84 \\
15 \\
26\end{array}$ & $\begin{array}{l}0.66 \\
23 \\
7\end{array}$ & & & & & & & & & & & & & & & & & 0.69 & -0.04 & $\begin{array}{c}0.03 \\
0.12 * *\end{array}$ \\
\hline
\end{tabular}

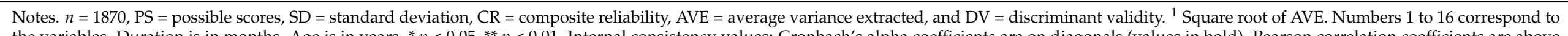

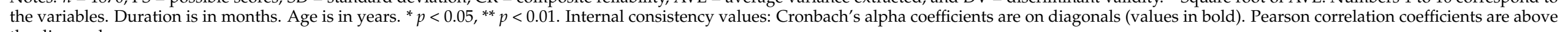
the diagonals. 


\subsection{Variables Associated with Study Abroad Difficulties and Its Subscales}

Hierarchical multiple regression analyses were conducted to reveal any significant associations for study abroad difficulties and its subscales: academic, leisure living, local viewpoints, daily living, responsive, and suppressive difficulties. Variables associated with the study abroad difficulties were entered using a three-step procedure. First, to control for possible effects, background demographic variables-age (in years), gender ( $0=$ female, $1=$ male), duration of stay (in months), and study abroad status $(0=$ short-term exchange, 1 = degree-seeking) - were entered into the equation. In the second step, after controlling for the background demographic variables, the various internet use subscales (online benefits, online habits, and online facilitation) were also entered into the equation. Lastly, in the third step, the big five personality traits (openness, conscientiousness, extraversion, agreeableness, and neuroticism) were entered into the equation.

Table 3 shows the results of the hierarchical multiple regression analyses. For study abroad difficulties as a whole, the control variables age $(\beta=0.098, t(1865)=4.159$, $p<0.001)$, duration of stay $(\beta=-0.065, t(1865)=-2.528, p<0.05)$, and status $(\beta=0.070$, $t(1865)=2.717, p<0.01)$ all showed significant associations and together explained $1.20 \%$ of the variance $(F[4,1865]=5.797, p<0.001)$. The internet use subscale online facilitation $(\beta=-0.163, t(1862)=-6.135, p<0.001)$ increases the explained variance to $5 \%(F[3,1862]$ $=25.096, p<0.001)$. Finally, agreeableness $(\beta=-0.127, t(1857)=-5.037, p<0.001)$ and neuroticism $(\beta=0.167, t(1857)=6.181, p<0.001)$ increased the explained variance to $12.30 \%(F[5,1857]=30.894, p<0.001)$.

Table 3. Hierarchical multiple regression analyses of study abroad difficulties.

\begin{tabular}{|c|c|c|c|c|c|c|c|c|}
\hline & Predictors & F Change & $t$ & df & B & SE & $\beta$ & $\mathbf{R}^{2}$ Change \\
\hline \multicolumn{9}{|c|}{ A. Dependent variable: Total study abroad difficulties } \\
\hline & Constant & & & & 1.986 & 0.059 & & \\
\hline \multirow{5}{*}{ I. } & Control variables & $5.797^{* * *}$ & & 4,1865 & & & & 0.012 \\
\hline & Age & & $4.159 * * *$ & & 0.009 & 0.002 & 0.098 & \\
\hline & Gender & & -0.120 & & -0.003 & 0.029 & -0.003 & \\
\hline & Duration of stay & & $-2.528 *$ & & -0.002 & 0.001 & -0.065 & \\
\hline & Status & & $2.717^{* *}$ & & 0.086 & 0.032 & 0.070 & \\
\hline \multirow{4}{*}{ II. } & Internet use & $25.096^{* * *}$ & & 3,1862 & & & & 0.038 \\
\hline & Online benefits & & -0.585 & & -0.011 & 0.018 & -0.016 & \\
\hline & Online habits & & -1.883 & & -0.034 & 0.018 & -0.052 & \\
\hline & $\begin{array}{c}\text { Online } \\
\text { facilitation }\end{array}$ & & $-6.135^{* * *}$ & & -0.120 & 0.020 & -0.163 & \\
\hline \multirow{6}{*}{ III. } & Personality & $30.894^{* * *}$ & & 5,1857 & & & & 0.073 \\
\hline & Openness & & -0.437 & & -0.011 & 0.025 & -0.010 & \\
\hline & Conscientiousness & & -0.857 & & -0.022 & 0.025 & -0.022 & \\
\hline & Extraversion & & -1.869 & & -0.046 & 0.025 & -0.047 & \\
\hline & Agreeableness & & $-5.037^{* * *}$ & & -0.138 & 0.027 & -0.127 & \\
\hline & Neuroticism & & $6.181^{* * *}$ & & 0.156 & 0.025 & 0.167 & \\
\hline \multicolumn{9}{|c|}{ B. Dependent variable: Academic difficulties } \\
\hline \multirow[t]{7}{*}{ I. } & Constant & & & & 2.049 & 0.080 & & \\
\hline & Control variables & $2.546^{*}$ & & 4,1865 & & & & 0.005 \\
\hline & Age & & 1.878 & & 0.006 & 0.003 & 0.045 & \\
\hline & Gender & & 0.022 & & 0.001 & 0.039 & 0.001 & \\
\hline & Duration of stay & & -0.332 & & 0.000 & 0.001 & -0.009 & \\
\hline & Status & & $2.562 * *$ & & 0.111 & 0.043 & 0.066 & \\
\hline & Internet use & $24.670 * * *$ & & 3,1862 & & & & 0.038 \\
\hline \multirow{3}{*}{ II. } & Online benefits & & -0.421 & & -0.010 & 0.025 & -0.011 & \\
\hline & Online habits & & -1.533 & & -0.038 & 0.025 & -0.042 & \\
\hline & $\begin{array}{c}\text { Online } \\
\text { facilitation }\end{array}$ & & $-6.405^{* * *}$ & & -0.171 & 0.027 & -0.170 & \\
\hline
\end{tabular}


Table 3. Cont.

\begin{tabular}{|c|c|c|c|c|c|c|c|c|}
\hline & Predictors & F Change & $t$ & df & B & SE & $\beta$ & $\mathbf{R}^{2}$ Change \\
\hline \multirow{6}{*}{ III. } & Personality & $24.683^{* * *}$ & & 5,1857 & & & & 0.060 \\
\hline & Openness & & -1.445 & & -0.050 & 0.034 & -0.035 & \\
\hline & Conscientiousness & & $-4.445^{* * *}$ & & -0.155 & 0.035 & -0.115 & \\
\hline & Extraversion & & $-2.725 * *$ & & -0.093 & 0.034 & -0.069 & \\
\hline & Agreeableness & & -1.197 & & -0.045 & 0.038 & -0.031 & \\
\hline & Neuroticism & & $4.057^{* * *}$ & & 0.141 & 0.035 & 0.111 & \\
\hline \multicolumn{9}{|c|}{ C. Dependent variable: Leisure living difficulties } \\
\hline & Constant & & & & 1.716 & 0.076 & & \\
\hline \multirow{5}{*}{ I. } & Control variables & $4.329 * *$ & & 4,1865 & & & & 0.009 \\
\hline & Age & & 2.177 * & & 0.006 & 0.003 & 0.052 & \\
\hline & Gender & & 1.169 & & 0.044 & 0.037 & 0.027 & \\
\hline & Duration of stay & & $-3.575^{* * *}$ & & -0.003 & 0.001 & -0.092 & \\
\hline & Status & & 1.768 & & 0.073 & 0.041 & 0.046 & \\
\hline \multirow{4}{*}{ II. } & Internet use & $25.168^{* * *}$ & & 3,1862 & & & & 0.039 \\
\hline & Online benefits & & 0.515 & & 0.012 & 0.023 & 0.014 & \\
\hline & Online habits & & -1.950 & & -0.046 & 0.023 & -0.054 & \\
\hline & $\begin{array}{c}\text { Online } \\
\text { facilitation }\end{array}$ & & $-6.680^{* * *}$ & & -0.171 & 0.026 & -0.177 & \\
\hline \multirow{6}{*}{ III. } & Personality & $14.785^{* * *}$ & & 5,1857 & & & & 0.036 \\
\hline & Openness & & -1.002 & & -0.033 & 0.033 & -0.024 & \\
\hline & Conscientiousness & & 0.690 & & 0.023 & 0.034 & 0.018 & \\
\hline & Extraversion & & -0.590 & & -0.019 & 0.033 & -0.015 & \\
\hline & Agreeableness & & $-3.702^{* * *}$ & & -0.136 & 0.037 & -0.096 & \\
\hline & Neuroticism & & $4.891^{* * *}$ & & 0.165 & 0.034 & 0.135 & \\
\hline \multicolumn{9}{|c|}{ D. Dependent variable: Local viewpoints difficulties } \\
\hline \multirow{7}{*}{ I. } & Constant & & & & 2.547 & 0.092 & & \\
\hline & Control variables & $3.490 * *$ & & 4,1865 & & & & 0.007 \\
\hline & Age & & $2.267 *$ & & 0.008 & 0.003 & 0.054 & \\
\hline & Gender & & -1.858 & & -0.083 & 0.045 & -0.043 & \\
\hline & Duration of stay & & $-2.824 * *$ & & -0.003 & 0.001 & -0.073 & \\
\hline & Status & & 1.581 & & 0.079 & 0.050 & 0.041 & \\
\hline & Internet use & $9.703^{* * *}$ & & 3,1862 & & & & 0.015 \\
\hline \multirow{3}{*}{ II. } & Online benefits & & $-3.928^{* * *}$ & & -0.112 & 0.029 & -0.108 & \\
\hline & Online habits & & -0.025 & & -0.001 & 0.029 & -0.001 & \\
\hline & $\begin{array}{c}\text { Online } \\
\text { facilitation }\end{array}$ & & -1.150 & & -0.036 & 0.031 & -0.031 & \\
\hline \multirow{6}{*}{ III. } & Personality & $12.264^{* * *}$ & & 5,1857 & & & & 0.031 \\
\hline & Openness & & -1.402 & & -0.057 & 0.041 & -0.035 & \\
\hline & Conscientiousness & & $-2.752^{* *}$ & & -0.113 & 0.041 & -0.073 & \\
\hline & Extraversion & & -0.828 & & -0.033 & 0.040 & -0.022 & \\
\hline & Agreeableness & & 0.050 & & 0.002 & 0.045 & 0.001 & \\
\hline & Neuroticism & & $4.163^{* * *}$ & & 0.171 & 0.041 & 0.117 & \\
\hline \multicolumn{9}{|c|}{ E. Dependent variable: Daily living difficulties } \\
\hline & Constant & & & & 1.706 & 0.088 & & \\
\hline \multirow{4}{*}{ I. } & Control variables & $8.104^{* * *}$ & & 4,1865 & & & & 0.017 \\
\hline & Age & & $5.201^{* * *}$ & & 0.017 & 0.003 & 0.123 & \\
\hline & Gender & & -0.688 & & -0.029 & 0.043 & -0.016 & \\
\hline & Duration of stay & & $-1.979 *$ & & -0.002 & 0.001 & -0.051 & \\
\hline \multirow{5}{*}{ II. } & Status & & $3.041^{* *}$ & & 0.144 & 0.047 & 0.078 & \\
\hline & Internet use & $6.071^{* * *}$ & & 3,1862 & & & & 0.010 \\
\hline & Online benefits & & 0.279 & & 0.008 & 0.027 & 0.008 & \\
\hline & Online habits & & -0.653 & & -0.018 & 0.027 & -0.018 & \\
\hline & $\begin{array}{c}\text { Online } \\
\text { facilitation }\end{array}$ & & $-3.485^{* * *}$ & & -0.104 & 0.030 & -0.094 & \\
\hline
\end{tabular}


Table 3. Cont.

\begin{tabular}{|c|c|c|c|c|c|c|c|c|}
\hline & Predictors & F Change & $t$ & df & B & SE & $\beta$ & $\mathbf{R}^{2}$ Change \\
\hline \multirow{6}{*}{ III. } & Personality & $24.903^{* * *}$ & & 5,1857 & & & & 0.061 \\
\hline & Openness & & 1.118 & & 0.043 & 0.038 & 0.027 & \\
\hline & Conscientiousness & & $3.193^{* * *}$ & & 0.123 & 0.039 & 0.083 & \\
\hline & Extraversion & & -1.158 & & -0.044 & 0.038 & -0.030 & \\
\hline & Agreeableness & & $-7.848^{* * *}$ & & -0.330 & 0.042 & -0.202 & \\
\hline & Neuroticism & & $4.452^{* * *}$ & & 0.172 & 0.039 & 0.123 & \\
\hline \multirow{8}{*}{ I. } & & & F. Dependent & ble: Resp & e difficul & & & \\
\hline & Constant & & & & 2.337 & 0.088 & & \\
\hline & Control variables & 1.150 & & 4,1865 & & & & 0.002 \\
\hline & Age & & 1.439 & & 0.005 & 0.003 & 0.034 & \\
\hline & Gender & & -0.208 & & -0.009 & 0.043 & -0.005 & \\
\hline & Duration of stay & & -1.449 & & -0.002 & 0.001 & -0.038 & \\
\hline & Status & & 1.633 & & 0.078 & 0.048 & 0.042 & \\
\hline & Internet use & $9.475^{* * *}$ & & 3,1862 & & & & 0.015 \\
\hline \multirow{3}{*}{ II. } & Online benefits & & 0.617 & & 0.017 & 0.027 & 0.017 & \\
\hline & Online habits & & $-1.984 *$ & & -0.054 & 0.027 & -0.056 & \\
\hline & $\begin{array}{c}\text { Online } \\
\text { facilitation }\end{array}$ & & $-3.652^{* * *}$ & & -0.109 & 0.030 & -0.098 & \\
\hline \multirow{8}{*}{ III. } & Personality & $22.949 * * *$ & & 5,1857 & & & & 0.057 \\
\hline & Openness & & 0.897 & & 0.034 & 0.038 & 0.022 & \\
\hline & Conscientiousness & & -0.413 & & -0.016 & 0.039 & -0.011 & \\
\hline & Extraversion & & $-3.248^{* * *}$ & & -0.123 & 0.038 & -0.084 & \\
\hline & Agreeableness & & $-4.391 * * *$ & & -0.186 & 0.042 & -0.114 & \\
\hline & Neuroticism & & $4.740^{* * *}$ & & 0.184 & 0.039 & 0.132 & \\
\hline & & & G. Dependent & ble: Supr & ve difficu & & & \\
\hline & Constant & & & & 1.559 & 0.075 & & \\
\hline \multirow{5}{*}{ I. } & Control variables & $6.797^{* * *}$ & & 4,1865 & & & & 0.014 \\
\hline & Age & & $4.681^{* * *}$ & & 0.013 & 0.003 & 0.111 & \\
\hline & Gender & & 1.536 & & 0.057 & 0.037 & 0.036 & \\
\hline & Duration of stay & & -0.362 & & 0.000 & 0.001 & -0.009 & \\
\hline & Status & & 0.773 & & 0.031 & 0.041 & 0.020 & \\
\hline \multirow{4}{*}{ II. } & Internet use & $15.231^{* * *}$ & & 3,1862 & & & & 0.024 \\
\hline & Online benefits & & 0.976 & & 0.023 & 0.023 & 0.027 & \\
\hline & Online habits & & $-2.001 *$ & & -0.047 & 0.023 & -0.055 & \\
\hline & $\begin{array}{c}\text { Online } \\
\text { facilitation }\end{array}$ & & $-5.109^{* * *}$ & & -0.130 & 0.025 & -0.136 & \\
\hline \multirow{6}{*}{ III. } & Personality & $7.402^{* * *}$ & & 5,1857 & & & & 0.019 \\
\hline & Openness & & -0.077 & & -0.003 & 0.033 & -0.002 & \\
\hline & Conscientiousness & & 0.217 & & 0.007 & 0.034 & 0.006 & \\
\hline & Extraversion & & 1.080 & & 0.036 & 0.033 & 0.028 & \\
\hline & Agreeableness & & $-3.697^{* * *}$ & & -0.136 & 0.037 & -0.097 & \\
\hline & Neuroticism & & $3.056 * *$ & & 0.103 & 0.034 & 0.086 & \\
\hline
\end{tabular}

Notes. $n=1870, t=$ for within-set predictors, $\mathrm{df}=$ degrees of freedom, $\mathrm{B}=$ unstandardized coefficients, $\mathrm{SE}=$ standard error, and $\beta=$ standardized coefficients. Age is in years. Gender: $0=$ female, $1=$ male. Duration of stay is in months. Status: $0=$ short-term exchange, $1=$ degree-seeking. ${ }^{*} p<0.05,{ }^{* *} p<0.01,{ }^{* * *} p<0.001$.

For the study abroad difficulties subscale academic difficulties, the only control variable that revealed a significant association was student status $(\beta=0.066, t(1865)=2.562$, $p<0.01)$, which explained $0.50 \%$ of the variance $(F[4,1865]=2.546, p<0.05)$. Next, the internet use subscale online facilitation $(\beta=-0.170, t(1862)=-6.405, p<0.001)$ increased the explained variance to $4.30 \%(F[3,1862]=24.670, p<0.001)$. Then, conscientiousness $(\beta=-0.115, t(1857)=-4.445, p<0.001)$ and neuroticism $(\beta=0.111, t(1857)=4.057$, $p<0.001)$ increased the explained variance to $10.30 \%(F[5,1857]=24.683, p<0.001)$.

For the study abroad difficulties subscale leisure living difficulties, the control variables age $(\beta=0.052, t(1865)=2.177, p<0.05)$ and duration of stay $(\beta=-0.092, t(1865)=$ $-3.575, p<0.001)$ revealed significant associations and explained $0.90 \%$ of the variance $(F[4,1865]=4.329, p<0.01)$. Next, the internet use subscale online facilitation $(\beta=-0.177$, 
$t(1862)=-6.680, p<0.001)$ increased the explained variance to $4.80 \%(F[3,1862]=25.168$, $p<0.001)$. Finally, agreeableness $(\beta=-0.096, t(1857)=-3.702, p<0.001)$ and neuroticism $(\beta=0.135, t(1857)=4.891, p<0.001)$ increased the explained variance to $8.40 \%(F[5,1857]$ $=14.785, p<0.001)$.

For the study abroad difficulties subscale local viewpoints, the control variables age $(\beta=0.054, t(1865)=2.267, p<0.05)$ and duration of stay $(\beta=-0.073, t(1865)=-2.824$, $p<0.01)$ revealed significant associations and explained $0.70 \%$ of the variance $(F[4,1865]=$ $3.490, p<0.01)$. The internet use subscale online benefits $(\beta=-0.108, t(1862)=-3.928$, $p<0.001)$ increased the explained variance to $2.20 \%(F[3,1862]=9.703, p<0.001)$, and conscientiousness $(\beta=-0.073, t(1857)=-2.752, p<0.01)$ and neuroticism $(\beta=0.117$, $t(1857)=4.163, p<0.001)$ increased the explained variance to $5.30 \%(F[5,1857]=12.264$, $p<0.001)$.

For the study abroad difficulties subscale daily living difficulties, the control variables age $(\beta=0.123, t(1865)=5.201, p<0.001)$, duration of stay $(\beta=-0.051, t(1865)=-1.979$, $p<0.05)$, and status $(\beta=0.078, t(1865)=3.041, p<0.01)$ all revealed significant associations and explained $1.70 \%$ of the variance $(F[4,1865]=8.104, p<0.001)$. The internet use subscale online facilitation $(\beta=-0.094, t(1862)=-3.485, p<0.001)$ increased the explained variance to $2.70 \%(F[3,1862]=6.071, p<0.001)$, and conscientiousness $(\beta=0.083, t(1857)=3.193$, $p<0.001)$, agreeableness $(\beta=-0.202, t(1857)=-7.848, p<0.001)$, and neuroticism $(\beta=0.123, t(1857)=4.452, p<0.001)$ further increased the explained variance to $8.80 \%$ $(F[5,1857]=24.903, p<0.001)$.

For the study abroad difficulties subscale responsive difficulties, none of the background demographics showed significant associations. The internet use subscales online habits $(\beta=-0.056, t(1862)=-1.984, p<0.05)$ and online facilitation $(\beta=-0.098, t(1862)$ $=-3.652, p<0.001)$ explained $1.70 \%$ of the variance $(F[3,1862]=9.475, p<0.001)$. Then, extraversion $(\beta=-0.084, t(1857)=-3.248, p<0.001)$, agreeableness $(\beta=-0.114, t(1857)$ $=-4.391, p<0.001)$, and neuroticism $(\beta=0.132, t(1857)=4.740, p<0.001)$ increased the explained variance to $7.40 \%(F[5,1857]=22.949, p<0.001)$.

Lastly, for the study abroad difficulties subscale suppressive difficulties, the only control variable with a significant association was age $(\beta=0.111, t(1865)=4.681, p<0.001)$, which explained $1.40 \%$ of the variance $(F[4,1865]=6.797, p<0.001)$. The internet use subscales online habits $(\beta=-0.055, t(1862)=-2.001, p<0.05)$ and online facilitation $(\beta=-0.136, t(1862)=-5.109, p<0.001)$ increased the explained variance to $3.80 \%(F[3$, $1862]=15.231, p<0.001)$, and agreeableness $(\beta=-0.097, t(1857)=-3.697, p<0.001)$ and neuroticism $(\beta=0.086, t(1857)=3.056, p<0.01)$ further increased the explained variance to $5.70 \%(F[5,1857]=7.402, p<0.001)$.

\subsection{Testing the Moderating Effect of Personality Traits}

To understand the moderating effect of the different personality traits, several moderation analyses were performed using Interaction! Software [75]. In addition to the moderation analyses, simple slopes difference tests were used to determine the three-way interactions within the moderated multiple regression models [78]. More specifically, the simple slopes difference tests were used to test the effects of extreme values [79]—high $(+2 \mathrm{SD})$ personality traits and their lower ( $-2 \mathrm{SD})$ counterparts - on the relationship between internet use and study abroad difficulties. For better interpretability of the results, all variables and predictors were standardized and centered prior to computing [80].

Table 4 shows the results of the moderation analysis and simple slopes models of study abroad difficulties, internet use, and openness. The total model accounted for $5.30 \%$ $(F[7,1862]=14.913, p<0.001)$ of the variance in study abroad difficulties. The results indicate that the control variables age $(\beta=0.074, p<0.01)$, duration of stay $(\beta=-0.074$, $p<0.01)$, and status $(\beta=0.081, p<0.01)$ significantly predicted study abroad difficulties. In addition, internet use $(\beta=-0.156, p<0.001)$, openness $(\beta=-0.078, p<0.01)$, and the interaction between internet use and openness $(\beta=-0.058, p<0.01)$ were statistically significant in the model. The effect size of the interaction was very small, with $\mathrm{f}^{2}=0.06$ [81] 
Simple slopes difference analysis showed that the relationship between internet use and study abroad difficulties was significant among high (slope $\beta=-0.272, p<0.001$ ) and low (slope $\beta=-0.040, p>0.05$, non-significant or ns) openness students $(\beta=-0.231$, $p<0.001$ [ [82]. Figure 2 shows the simple slope plot for the moderation effect of openness, which signifies that openness strengthens the negative relationship between internet use and study abroad difficulties.

Table 4. Moderation analysis and simple slopes models of study abroad difficulties, internet use, and openness.

\begin{tabular}{|c|c|c|c|c|c|c|}
\hline Full Regression Model & $\beta$ & SE & $t$ & $p$ & LLCI & ULCI \\
\hline \multicolumn{7}{|l|}{ Predictor variables } \\
\hline Constant & 0.013 & 0.023 & 0.58 & 0.563 & -0.032 & 0.059 \\
\hline \multicolumn{7}{|l|}{ Covariates } \\
\hline Age & 0.074 & 0.023 & 3.15 & 0.002 & 0.028 & 0.120 \\
\hline Gender & -0.006 & 0.023 & -0.27 & 0.789 & -0.051 & 0.039 \\
\hline Duration of stay & -0.074 & 0.025 & -2.90 & 0.004 & -0.123 & -0.024 \\
\hline Status & 0.081 & 0.025 & 3.21 & 0.001 & 0.032 & 0.131 \\
\hline \multicolumn{7}{|l|}{ Main effects } \\
\hline Internet use & -0.156 & 0.024 & -6.63 & $<0.001$ & -0.202 & -0.110 \\
\hline Openness & -0.078 & 0.023 & -3.33 & 0.001 & -0.123 & -0.032 \\
\hline \multicolumn{7}{|l|}{ Two-way interaction } \\
\hline Internet use X Openness & -0.058 & 0.022 & -2.65 & 0.008 & -0.101 & -0.015 \\
\hline \multirow[t]{2}{*}{ Model fit } & $\mathbf{R}^{2}$ & Adjusted $\mathbf{R}^{2}$ & $\mathbf{f}^{2}$ & & & \\
\hline & 0.053 & 0.050 & 0.06 & & & \\
\hline Simple slopes models & $\beta$ & SE & $t$ & $p$ & & \\
\hline \multicolumn{7}{|l|}{ Groupings } \\
\hline \multicolumn{7}{|l|}{$+2 \mathrm{SD}(n=27)$} \\
\hline Intercept & -0.142 & & & & & \\
\hline Slope & -0.272 & 0.055 & -4.94 & $<0.001$ & -0.380 & -0.164 \\
\hline \multicolumn{7}{|l|}{ Mean $(n=1807)$} \\
\hline Intercept & 0.013 & & & & & \\
\hline Slope & -0.156 & 0.024 & -6.63 & $<0.001$ & -0.202 & -0.110 \\
\hline \multicolumn{7}{|l|}{$-2 \mathrm{SD}(n=36)$} \\
\hline Intercept & 0.168 & & & & & \\
\hline Slope & -0.040 & 0.050 & -0.81 & 0.416 & -0.138 & 0.057 \\
\hline \multicolumn{7}{|l|}{$\begin{array}{l}\text { Simple slopes difference } \\
\qquad(+2 \mathrm{SD},-2 \mathrm{SD})\end{array}$} \\
\hline & -0.231 & 0.027 & -8.63 & $<0.001$ & & \\
\hline
\end{tabular}

Notes. All variables and predictors were standardized and centered prior to computing. $n=1870$. $\beta=$ standardized coefficients, SE $=$ standard error, $\mathrm{LLCI}=$ lower level confidence interval, and ULCI $=$ upper level confidence interval. Age is in years. Gender: $0=$ female, $1=$ male. Duration of stay is in months. Status: $0=$ short-term exchange, $1=$ degree-seeking.

Table 5 shows the results of the moderation analysis and simple slopes models of study abroad difficulties, internet use, and conscientiousness. The total model accounted for $6.51 \%(F[7,1862]=18.511, p<0.001)$ of the variance in study abroad difficulties. The results indicate that the control variables age $(\beta=0.067, p<0.01)$, duration of stay $(\beta=-0.075$, $p<0.01)$, and status $(\beta=0.088, p<0.001)$ significantly predicted study abroad difficulties. In addition, internet use $(\beta=-0.143, p<0.001)$ and conscientiousness $(\beta=-0.143$, $p<0.001$ ) were statistically significant, although the interaction between internet use and conscientiousness ( $\beta=-0.040, p>0.05$, ns) was not statistically significant in the model. Simple slopes difference analysis showed that the relationship between internet use and study abroad difficulties was significant among high (slope $\beta=-0.223, p<0.001$ ) and low (slope $\beta=-0.062, p>0.05$, ns) conscientiousness students $(\beta=-0.161, p<0.001)$. Figure 3 shows the simple slope plot for the moderation effect of conscientiousness, signifying that conscientiousness strengthens the negative relationship between internet use and study abroad difficulties. 


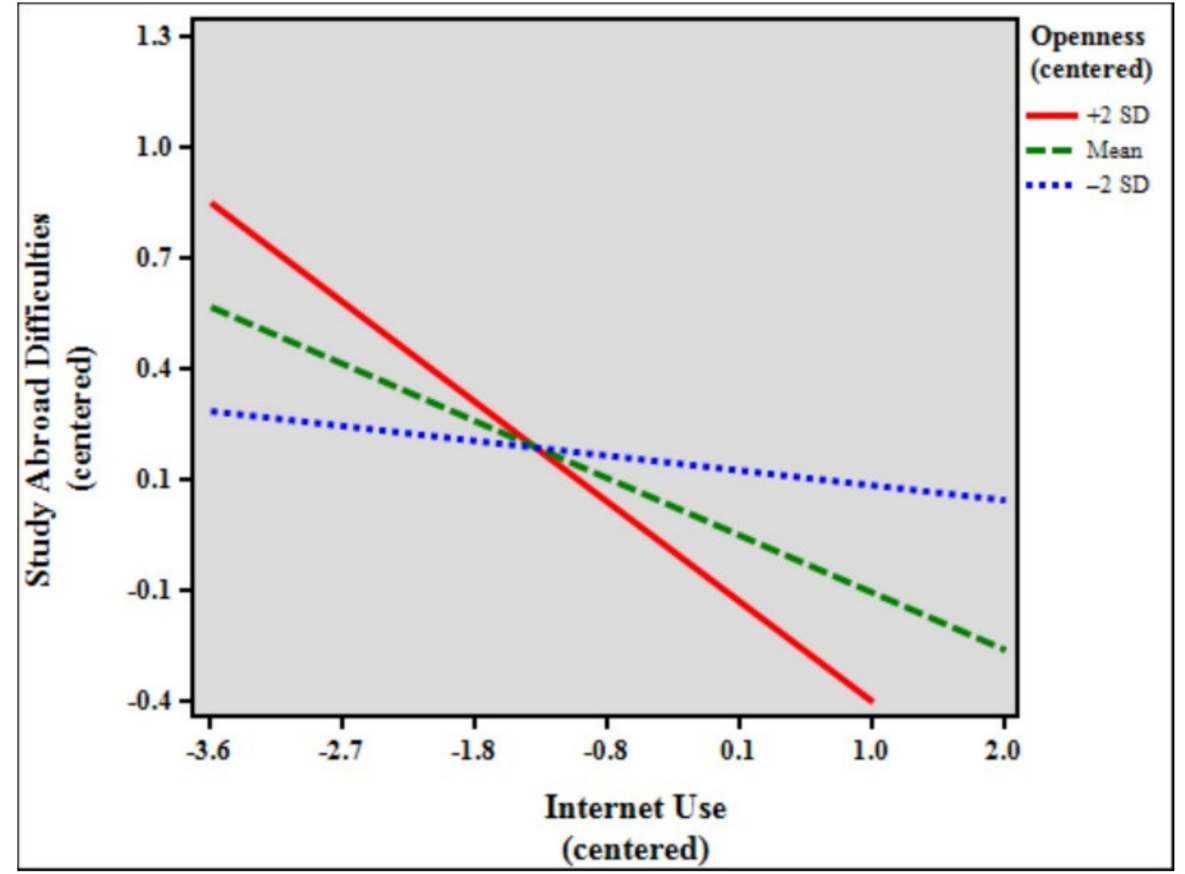

Figure 2. Simple slope plot for the moderation effect of openness.

Table 5. Moderation analysis and simple slopes models of study abroad difficulties, internet use, and conscientiousness.

\begin{tabular}{|c|c|c|c|c|c|c|}
\hline Full Regression Model & $\beta$ & SE & $t$ & $p$ & LLCI & ULCI \\
\hline \multicolumn{7}{|l|}{ Predictor variables } \\
\hline Constant & 0.008 & 0.023 & 0.34 & 0.733 & -0.037 & 0.053 \\
\hline \multicolumn{7}{|l|}{ Covariates } \\
\hline Age & 0.067 & 0.023 & 2.86 & 0.004 & 0.021 & 0.112 \\
\hline Gender & 0.001 & 0.023 & 0.05 & 0.963 & -0.044 & 0.046 \\
\hline Duration of stay & -0.075 & 0.025 & -2.98 & 0.003 & -0.125 & -0.026 \\
\hline Status & 0.088 & 0.025 & 3.51 & $<0.001$ & 0.039 & 0.138 \\
\hline \multicolumn{7}{|l|}{ Main effects } \\
\hline Internet use & -0.143 & 0.024 & -6.06 & $<0.001$ & -0.189 & -0.097 \\
\hline Conscientiousness & -0.143 & 0.023 & -6.20 & 0.001 & -0.188 & -0.098 \\
\hline \multicolumn{7}{|l|}{ Two-way interaction } \\
\hline $\begin{array}{c}\text { Internet use } X \\
\text { Conscientiousness }\end{array}$ & -0.040 & 0.022 & -1.81 & 0.070 & -0.188 & 0.003 \\
\hline \multirow[t]{2}{*}{ Model fit } & $\mathbf{R}^{2}$ & Adjusted $\mathbf{R}^{2}$ & $\mathbf{f}^{2}$ & & & \\
\hline & 0.065 & 0.062 & 0.07 & & & \\
\hline Simple slopes models & $\beta$ & SE & $t$ & $p$ & & \\
\hline \multicolumn{7}{|l|}{ Groupings } \\
\hline \multicolumn{7}{|l|}{$+2 \operatorname{SD}(n=48)$} \\
\hline Intercept & -0.278 & & & & & \\
\hline Slope & -0.223 & 0.054 & -4.10 & $<0.001$ & -0.330 & -0.117 \\
\hline \multicolumn{7}{|l|}{ Mean $(n=1781)$} \\
\hline Intercept & 0.008 & & & & & \\
\hline Slope & -0.143 & 0.024 & -6.06 & $<0.001$ & -0.189 & -0.097 \\
\hline \multicolumn{7}{|l|}{$-2 \mathrm{SD}(n=41)$} \\
\hline Intercept & 0.294 & & & & & \\
\hline Slope & -0.062 & 0.050 & -1.25 & 0.213 & -0.159 & 0.036 \\
\hline \multicolumn{7}{|l|}{$\begin{array}{l}\text { Simple slopes difference } \\
\qquad(+2 \mathrm{SD},-2 \mathrm{SD})\end{array}$} \\
\hline & -0.161 & 0.027 & -6.04 & $<0.001$ & & \\
\hline
\end{tabular}

Notes. All variables and predictors were standardized and centered prior to computing. $n=1870$. $\beta=$ standardized coefficients, $\mathrm{SE}=$ standard error, $\mathrm{LLCI}=$ lower level confidence interval, and ULCI = upper level confidence interval. Age is in years. Gender: $0=$ female, $1=$ male. Duration of stay is in months. Status: $0=$ short-term exchange, $1=$ degree-seeking. 


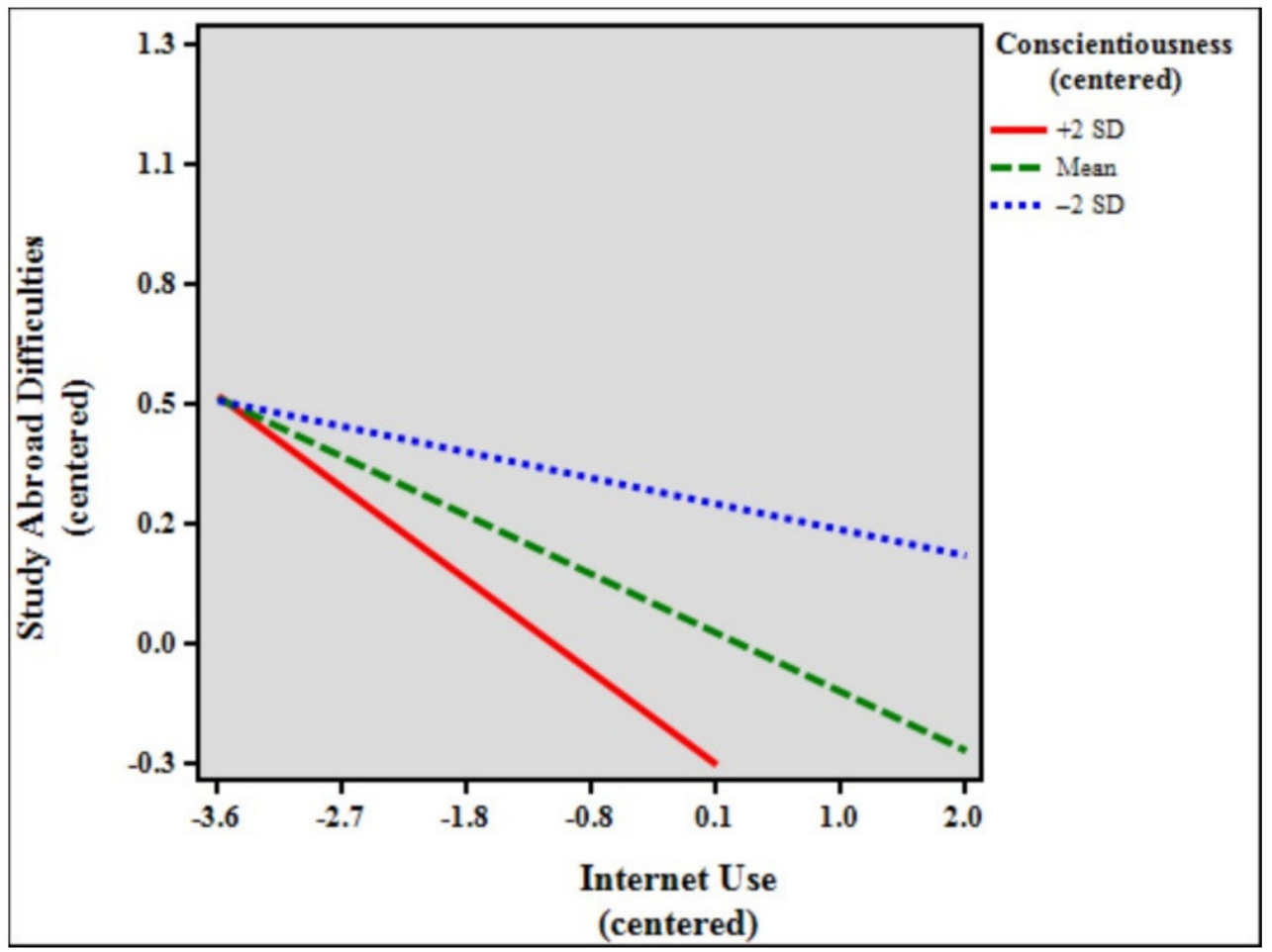

Figure 3. Simple slope plot for the moderation effect of conscientiousness.

Table 6 shows the results of the moderation analysis and simple slopes models of study abroad difficulties, internet use, and extraversion. The total model accounted for $7.15 \%(F[7,1862]=20.471, p<0.001)$ of the variance in study abroad difficulties. The results indicate that the control variables age $(\beta=0.072, p<0.01)$, duration of stay $(\beta=-0.073$, $p<0.01)$, and status $(\beta=0.075, p<0.01)$ significantly predicted study abroad difficulties. In addition, internet use $(\beta=-0.137, p<0.001)$, extraversion $(\beta=-0.151, p<0.001)$, and the interaction between internet use and extraversion $(\beta=-0.073, p<0.001)$ were statistically significant in the model. The effect size of the interaction was very small, with $\mathrm{f}^{2}=0.08$. Simple slopes difference analysis showed that the relationship between internet use and study abroad difficulties was significant among high (slope $\beta=-0.283, p<0.001$ ) and low (slope $\beta=0.010, p>0.05$, ns) extraversion students $(\beta=-0.293, p<0.001)$. Figure 4 shows the simple slope plot for the moderation effect of extraversion, signifying that extraversion strengthens the negative relationship between internet use and study abroad difficulties.

Table 6. Moderation analysis and simple slopes models of study abroad difficulties, internet use, and extraversion.

\begin{tabular}{|c|c|c|c|c|c|c|}
\hline Full Regression Model & $\beta$ & SE & $t$ & $p$ & LLCI & ULCI \\
\hline \multicolumn{7}{|l|}{ Predictor variables } \\
\hline Constant & 0.017 & 0.023 & 0.74 & 0.462 & -0.028 & 0.062 \\
\hline \multicolumn{7}{|l|}{ Covariates } \\
\hline Age & 0.072 & 0.023 & 3.09 & 0.002 & 0.026 & 0.117 \\
\hline Gender & -0.008 & 0.023 & -0.38 & 0.707 & -0.053 & 0.036 \\
\hline Duration of stay & -0.073 & 0.025 & -2.91 & 0.004 & -0.122 & -0.024 \\
\hline Status & 0.075 & 0.025 & 3.01 & 0.003 & 0.026 & 0.124 \\
\hline \multicolumn{7}{|l|}{ Main effects } \\
\hline Internet use & -0.137 & 0.023 & -5.86 & $<0.001$ & -0.182 & -0.091 \\
\hline Extraversion & -0.151 & 0.023 & -6.56 & $<0.001$ & -0.196 & -0.106 \\
\hline Two-way interaction & & & & & & \\
\hline Internet use $X$ Extraversion & -0.073 & 0.021 & -3.50 & $<0.001$ & -0.114 & -0.032 \\
\hline
\end{tabular}


Table 6. Cont.

\begin{tabular}{|c|c|c|c|c|c|c|}
\hline Full Regression Model & $\beta$ & SE & $t$ & $p$ & LLCI & ULCI \\
\hline Model fit & $\begin{array}{c}\mathbf{R}^{\mathbf{2}} \\
0.071\end{array}$ & $\begin{array}{c}\text { Adjusted } \mathbf{R}^{2} \\
0.068\end{array}$ & $\begin{array}{c}\mathbf{f}^{2} \\
0.08\end{array}$ & & & \\
\hline Simple slopes models & $\beta$ & SE & $t$ & $p$ & & \\
\hline $\begin{array}{c}\text { Groupings } \\
+2 \mathrm{SD}(n=47) \\
\text { Intercept } \\
\text { Slope }\end{array}$ & $\begin{array}{l}-0.285 \\
-0.283\end{array}$ & 0.054 & -5.20 & $<0.001$ & -0.390 & -0.177 \\
\hline $\begin{array}{c}\text { Mean }(n=1782) \\
\text { Intercept }\end{array}$ & 0.017 & & & & & \\
\hline $\begin{array}{c}\text { Slope } \\
-2 \text { SD }(n=41) \\
\text { Intercept }\end{array}$ & -0.137 & 0.023 & -5.86 & $<0.001$ & -0.182 & -0.091 \\
\hline $\begin{array}{c}\text { Slope } \\
\text { Simple slopes difference } \\
(+2 \text { SD },-2 \text { SD })\end{array}$ & 0.010 & 0.049 & -11.02 & 0.840 & -0.087 & 0.106 \\
\hline
\end{tabular}

Notes. All variables and predictors were standardized and centered prior to computing. $n=1870 . \quad \beta=$ standardized coefficients, $\mathrm{SE}=$ standard error, $\mathrm{LLCI}=$ lower level confidence interval, and ULCI $=$ upper level confidence interval. Age is in years. Gender: $0=$ female, $1=$ male. Duration of stay is in months. Status: $0=$ short-term exchange, $1=$ degree-seeking.

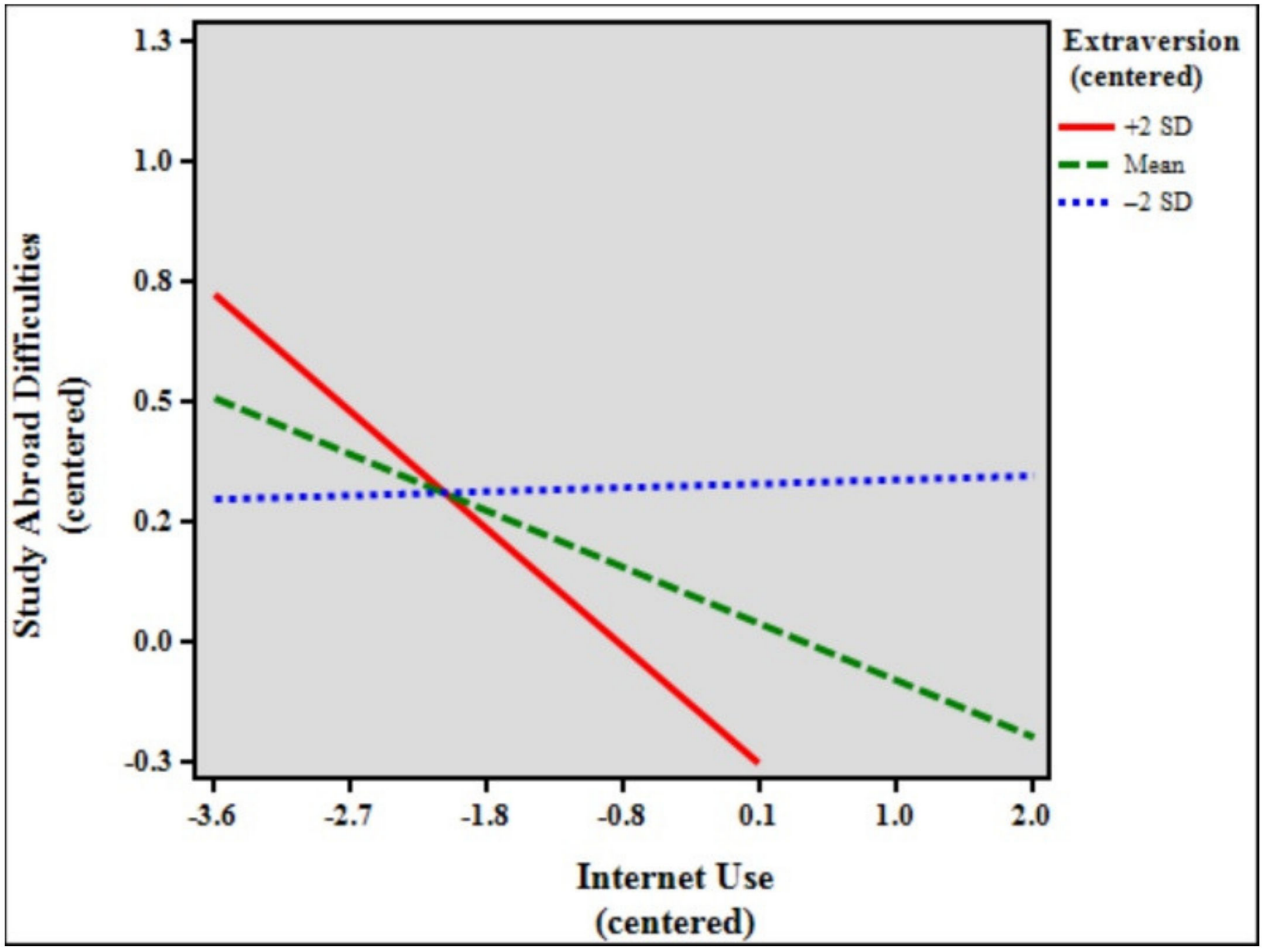

Figure 4. Simple slope plot for the moderation effect of extraversion.

Table 7 shows the results of the moderation analysis and simple slopes models of study abroad difficulties, internet use, and agreeableness. The total model accounted for $9.06 \%(F[7,1862]=26.512, p<0.001)$ of the variance in study abroad difficulties. The results indicate that the control variables age $(\beta=0.068, p<0.01)$, duration of stay $(\beta=-0.074$, $p<0.01)$, and status $(\beta=0.094, p<0.001)$ significantly predicted study abroad difficulties. In addition, internet use $(\beta=-0.125, p<0.001)$, agreeableness $(\beta=-0.216, p<0.001)$, and the interaction between internet use and agreeableness $(\beta=-0.058, p<0.01)$ were statistically significant in the model. The effect size of the interaction was very small, with $\mathrm{f}^{2}=0.10$. Simple slopes difference analysis showed that the relationship between internet 
use and study abroad difficulties was significant among high (slope $\beta=-0.242, p<0.001$ ) and low (slope $\beta=-0.008, p>0.05, \mathrm{~ns})$ agreeableness students $(\beta=-0.234, p<0.001$ ). Figure 5 shows the simple slope plot for the moderation effect of agreeableness, signifying that agreeableness strengthens the negative relationship between internet use and study abroad difficulties.

Table 7. Moderation analysis and simple slopes models of study abroad difficulties, internet use, and agreeableness.

\begin{tabular}{|c|c|c|c|c|c|c|}
\hline Full Regression Model & $\beta$ & SE & $t$ & $p$ & LLCI & ULCI \\
\hline \multicolumn{7}{|l|}{ Predictor variables } \\
\hline Constant & 0.013 & 0.023 & 0.59 & 0.556 & -0.031 & 0.058 \\
\hline \multicolumn{7}{|l|}{ Covariates } \\
\hline Age & 0.068 & 0.023 & 2.97 & 0.003 & 0.023 & 0.113 \\
\hline Gender & -0.011 & 0.022 & -0.50 & 0.617 & -0.055 & 0.033 \\
\hline Duration of stay & -0.074 & 0.025 & -3.00 & 0.003 & -0.123 & -0.026 \\
\hline Status & 0.094 & 0.025 & 3.80 & $<0.001$ & 0.046 & 0.143 \\
\hline \multicolumn{7}{|l|}{ Main effects } \\
\hline Internet use & -0.125 & 0.023 & -5.42 & $<0.001$ & -0.170 & -0.080 \\
\hline Agreeableness & -0.216 & 0.023 & -9.47 & $<0.001$ & -0.261 & -0.171 \\
\hline \multicolumn{7}{|l|}{ Two-way interaction } \\
\hline $\begin{array}{l}\text { Internet use X } \\
\text { Agreeableness }\end{array}$ & -0.058 & 0.021 & -2.75 & 0.006 & -0.100 & -0.017 \\
\hline \multirow[t]{2}{*}{ Model fit } & $\mathbf{R}^{2}$ & Adjusted $R^{2}$ & $\mathbf{f}^{2}$ & & & \\
\hline & 0.091 & 0.087 & 0.10 & & & \\
\hline Simple slopes models & $\beta$ & SE & $t$ & $p$ & & \\
\hline \multicolumn{7}{|l|}{ Groupings } \\
\hline \multicolumn{7}{|l|}{$+2 \mathrm{SD}(n=39)$} \\
\hline Intercept & -0.419 & & & & & \\
\hline Slope & -0.242 & 0.054 & -4.50 & $<0.001$ & -0.347 & -0.136 \\
\hline \multicolumn{7}{|l|}{ Mean $(n=1789)$} \\
\hline Intercept & 0.013 & & & & & \\
\hline Slope & -0.125 & 0.023 & -5.42 & $<0.001$ & -0.170 & -0.080 \\
\hline \multicolumn{7}{|l|}{$-2 \mathrm{SD}(n=42)$} \\
\hline Intercept & 0.445 & & & & & \\
\hline Slope & -0.008 & 0.049 & -0.16 & 0.872 & -0.104 & 0.088 \\
\hline \multicolumn{7}{|l|}{$\begin{array}{l}\text { Simple slopes difference } \\
\qquad(+2 \mathrm{SD},-2 \mathrm{SD})\end{array}$} \\
\hline & -0.234 & 0.026 & -8.92 & $<0.001$ & & \\
\hline
\end{tabular}

Notes. All variables and predictors were standardized and centered prior to computing. $n=1870$. $\beta=$ standardized coefficients, $\mathrm{SE}=$ standard error, LLCI = lower level confidence interval, and ULCI = upper level confidence interval. Age is in years. Gender: $0=$ female, $1=$ male. Duration of stay is in months. Status: $0=$ short-term exchange, $1=$ degree-seeking.

Table 8 shows the results of the moderation analysis and simple slopes models of study abroad difficulties, internet use, and neuroticism. The total model accounted for $10.60 \%$ $(F[7,1862]=31.551, p<0.001)$ of the variance in study abroad difficulties. The results indicate that the control variables age $(\beta=0.072, p<0.01)$, duration of stay $(\beta=-0.069$, $p<0.01)$, and status $(\beta=0.097, p<0.001)$ significantly predicted study abroad difficulties. In addition, internet use $(\beta=-0.123, p<0.001)$, neuroticism $(\beta=0.242, p<0.001)$, and the interaction between internet use and neuroticism $(\beta=0.060, p<0.01)$ were statistically significant in the model. The effect size of the interaction was very small, with $\mathrm{f}^{2}=0.12$. Simple slopes difference analysis showed that the relationship between internet use and study abroad difficulties was significant among high (slope $\beta=-0.003, p>0.05$, ns) and low (slope $\beta=-0.242, p<0.001)$ neuroticism students $(\beta=0.239, p<0.001)$. Figure 6 shows the simple slope plot for the moderation effect of neuroticism, signifying that neuroticism dampens the negative relationship between internet use and study abroad difficulties. 


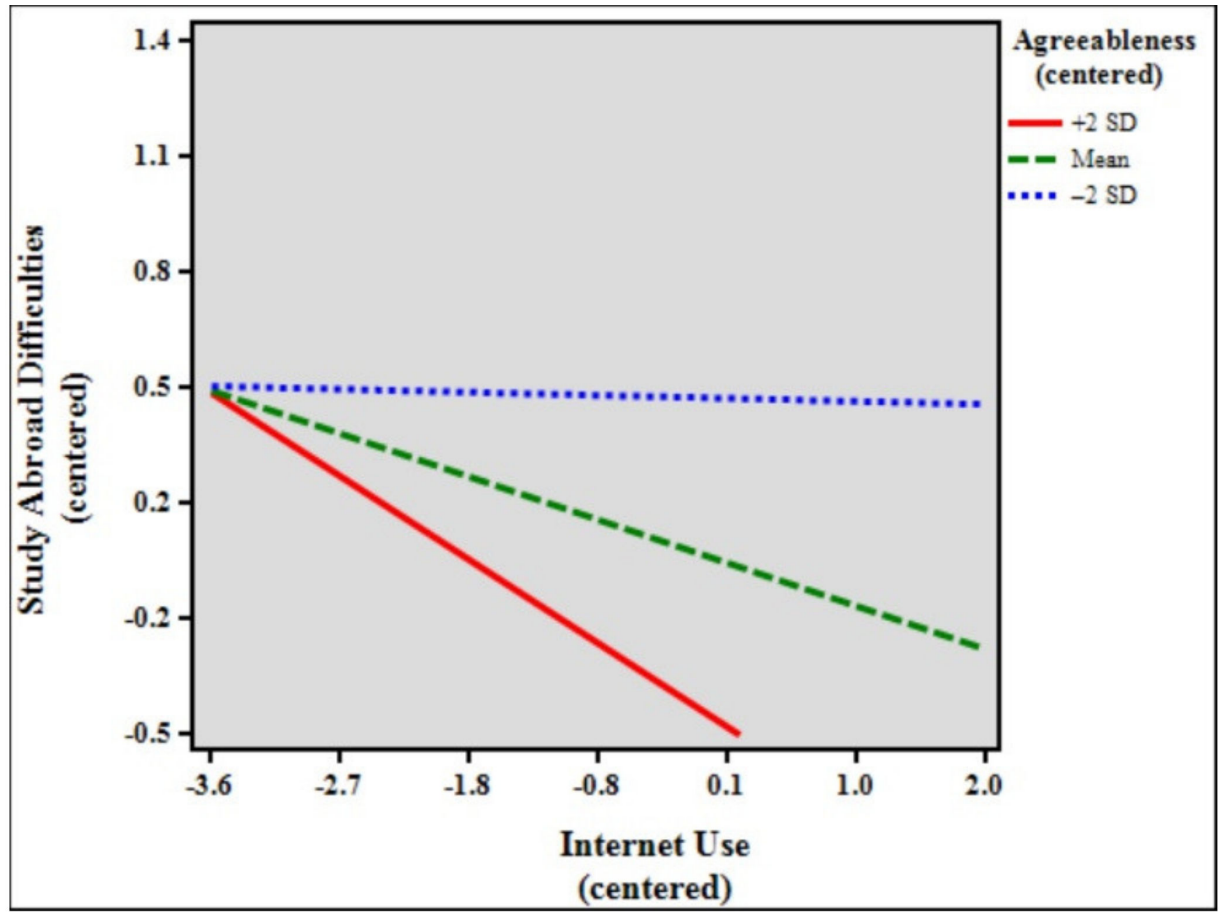

Figure 5. Simple slope plot for the moderation effect of agreeableness.

Table 8. Moderation analysis and simple slopes models of study abroad difficulties, internet use, and neuroticism.

\begin{tabular}{|c|c|c|c|c|c|c|}
\hline Full Regression Model & $\beta$ & SE & $t$ & $p$ & LLCI & ULCI \\
\hline \multicolumn{7}{|l|}{ Predictor variables } \\
\hline Constant & 0.011 & 0.022 & 0.50 & 0.618 & -0.033 & 0.055 \\
\hline \multicolumn{7}{|l|}{ Covariates } \\
\hline Age & 0.072 & 0.023 & 3.18 & 0.001 & 0.028 & 0.117 \\
\hline Gender & 0.006 & 0.022 & 0.27 & 0.787 & -0.038 & 0.050 \\
\hline Duration of stay & -0.069 & 0.025 & -2.81 & 0.005 & -0.117 & -0.021 \\
\hline Status & 0.097 & 0.025 & 3.93 & $<0.001$ & 0.048 & 0.145 \\
\hline \multicolumn{7}{|l|}{ Main effects } \\
\hline Internet use & -0.123 & 0.023 & -5.37 & $<0.001$ & -0.167 & -0.078 \\
\hline Neuroticism & 0.242 & 0.023 & 10.69 & $<0.001$ & 0.197 & 0.286 \\
\hline \multicolumn{7}{|l|}{ Two-way interaction } \\
\hline Internet use X Neuroticism & 0.060 & 0.022 & 2.71 & 0.007 & 0.017 & 0.103 \\
\hline \multirow[t]{2}{*}{ Model fit } & $\mathbf{R}^{2}$ & Adjusted $\mathrm{R}^{2}$ & $\mathbf{f}^{2}$ & & & \\
\hline & 0.106 & 0.103 & 0.12 & & & \\
\hline Simple slopes models & $\beta$ & SE & $t$ & $p$ & & \\
\hline \multicolumn{7}{|l|}{ Groupings } \\
\hline \multicolumn{7}{|l|}{$+2 \operatorname{SD}(n=24)$} \\
\hline Intercept & 0.495 & & & & & \\
\hline Slope & -0.003 & 0.053 & -0.06 & 0.955 & -0.108 & 0.101 \\
\hline \multicolumn{7}{|l|}{ Mean $(n=1790)$} \\
\hline Intercept & 0.011 & & & & & \\
\hline Slope & -0.123 & 0.023 & -5.37 & $<0.001$ & -0.167 & -0.078 \\
\hline \multicolumn{7}{|l|}{$-2 \mathrm{SD}(n=56)$} \\
\hline Intercept & -0.473 & & & & & \\
\hline Slope & -0.242 & 0.048 & -5.02 & $<0.001$ & -0.337 & -0.147 \\
\hline \multicolumn{7}{|l|}{$\begin{array}{l}\text { Simple slopes difference } \\
\qquad(+2 \mathrm{SD},-2 \mathrm{SD})\end{array}$} \\
\hline & 0.239 & 0.026 & 9.37 & $<0.001$ & & \\
\hline
\end{tabular}

Notes. All variables and predictors were standardized and centered prior to computing. $n=1870$. $\beta=$ standardized coefficients, $\mathrm{SE}=$ standard error, $\mathrm{LLCI}=$ lower level confidence interval, and ULCI $=$ upper level confidence interval. Age is in years. Gender: $0=$ female, $1=$ male. Duration of stay is in months. Status: $0=$ short-term exchange, $1=$ degree-seeking. 


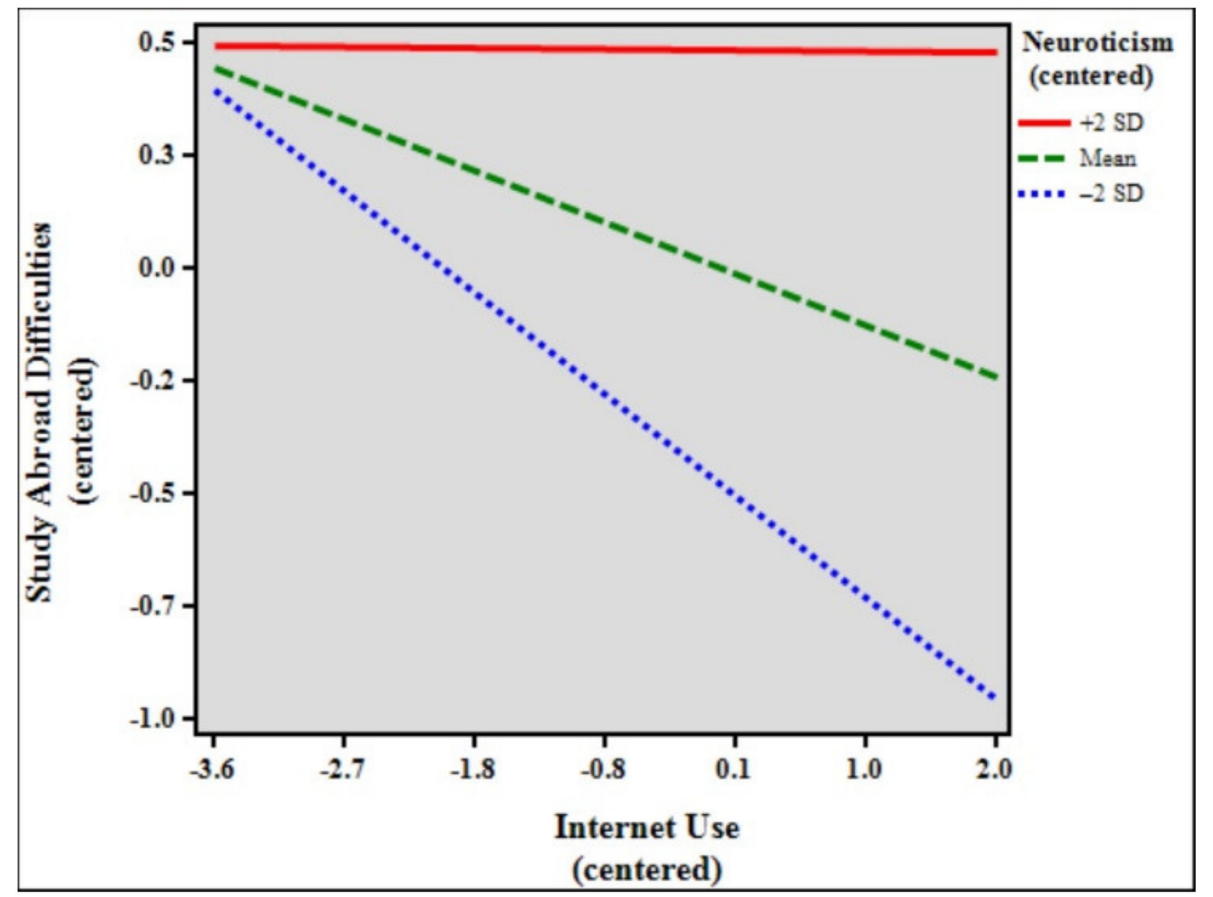

Figure 6. Simple slope plot for the moderation effect of neuroticism.

\section{Discussion}

The primary objective of the current study was to examine the moderating effects of personality traits on the relationship between internet use and study abroad difficulties. To achieve this, several analyses were performed. The descriptive statistics show that local viewpoints $(M=2.69, S D=0.96)$ ranked highest among the difficulties faced by study abroad students. Local viewpoints are issues related to understanding the Taiwanese world view and local perspectives on cultural issues and seeing things from a Taiwanese perspective [74]. Gaining insights into the local perspective is an important component of cross-cultural understanding and awareness [83]. Although participating in study abroad programs can help develop an individual's cross-cultural awareness [84], the key to building cross-cultural understanding is still actual physical interaction with host country nationals $[85,86]$. Local immersion can often be difficult, but cultural understanding is needed to gain insight into local values [87]. Study abroad students must have a desire to interact with host nationals (in the current case, Taiwanese people) [88], as without this, cross-cultural understanding and awareness will be difficult to achieve.

The results also show that leisure living $(\mathrm{M}=1.88, \mathrm{SD}=0.80)$ ranks lowest among the difficulties. Leisure living refers to issues pertaining to the cultural tourism aspect of studying abroad. Low perceived difficulties are noted on issues that relate to sightseeing, shopping, buying groceries, eating in restaurants, and using the mass transportation system [74]. The concept of educational or cultural tourism is an approach in study abroad that serves as a starting point for more in-depth and meaningful interaction with host nationals $[89,90]$. The current results echo previous findings, which have highlighted the importance of local tourism as one of the major pull factors for study abroad students in Taiwan [91].

Regarding the study abroad students' internet use motives, online facilitation $(\mathrm{M}=3.81$, $\mathrm{SD}=0.83$ ) ranked highest. Online facilitation refers to issues relating to students' use of the internet to facilitate social and cultural activities [27]. This is related to the area of leisure living, as students tend to use the internet to find information on places to visit, cultural events to attend, and other leisure activities. More importantly, all the internet use subscales were significantly and negatively correlated with all the study abroad difficulties subscales (except for the correlation between daily living and online benefits, which is not significant), signifying that internet use somehow minimizes study abroad difficulties, as 
noted in previous studies [27,92]. For instance, in the current study, online benefits are regarded as factors that can help reduce both social and academic difficulties, while online habits-or, more specifically, social media habits—-help students stay connected with their family and friends. In essence, the findings suggest that internet use has positive effects in reducing study abroad difficulties.

The personality traits of students were also collected, with agreeableness $(\mathrm{M}=3.57$, $\mathrm{SD}=0.56)$ ranking as the most evident, openness $(\mathrm{M}=3.34, \mathrm{SD}=0.58)$ coming second, and neuroticism $(\mathrm{M}=2.84, \mathrm{SD}=0.66)$ last. This finding supports the results of a previous study conducted in Germany, which found that students who participated in study abroad programs scored higher for agreeableness and openness [42]. Interestingly, correlational analyses showed that neuroticism was significantly and negatively correlated with the other personality traits and with all the internet use subscales. Importantly, neuroticism is significantly and positively correlated with all the study abroad difficulties subscales. This implies that students who rate high for neuroticism tend to have more study abroad difficulties and less internet use. This finding aligns with Kong's [93] suggestion that neuroticism is related to study abroad anxiety and affects an individual's sociocultural adaptation [94].

As for the duration of stay, the findings show negative correlations with leisure living and local viewpoints difficulties, as well as with online benefits and online habits. These are expected and signify that the longer a student stays in Taiwan, the more acquainted they become with host nationals and, hence, the fewer local viewpoints difficulties they experience. Researchers have pointed out that longer duration of stay increases the chances of interaction with host nationals $[95,96]$ and, hence, contributes to a better understanding of the local culture and values. At the same time, the longer a student remains in Taiwan, the more opportunities they have to travel around the country. Furthermore, students who spent less time in Taiwan had significantly higher perceived online benefits and habits. The findings are also not surprising, as they suggest that students who are new to studying abroad (those who have spent less time in Taiwan) tend to be more engaged in their academic work and are more likely to be more connected with their family and friends.

The findings also show that younger students tend to have difficulties with daily living and with suppressing their emotions. More specifically, younger students seem to have greater difficulty adjusting to the local etiquette and student life in Taiwan. In addition, they might be unable to cope with the many affective changes that are linked to living and studying in a foreign country. This aligns somewhat with a study on Korean study abroad students, which found that younger students were more reluctant to seek help from others with their adjustment difficulties [97]. Lastly, the findings show that older students tend to be less adept at using the internet. Although it has been suggested that older individuals tend to refrain from technology use, however, this typically applies to those over 65 years of age [98]. In fact, some researchers have proposed that the perceived usefulness and intention regarding internet use do not change with age [99].

After the descriptive analyses were completed, the various variables were assessed for gender differences. An independent samples $t$-test showed that male students had greater difficulty in suppressing their emotions than their female counterparts. This finding is rather unique, as most previous studies have found that male students are more emotionally stable than female students [36,100]. Gender differences were also found for neuroticism, with female students rating slightly higher than male students. Since neuroticism is related to emotional stability [101], so it follows that female students would be more emotionally unstable than men. Gender differences were also found in online facilitation, whereby female students were more likely to use the internet to facilitate their social and cultural activities than male students. In addition, male students were also found to be more conscientious than female students.

Additional independent samples $t$-tests were performed on study abroad students' status. The findings show that degree-seeking students rated significantly higher for academic and daily living difficulties. Academic difficulties are issues related to the teaching 
and learning processes during lectures and fulfilling school work. This finding coincides with those of numerous previous researchers, who have reported that academic stress and pressure to succeed are experienced by Asian students studying in the United States and Western students studying in China $[29,102,103]$. Similarly, significant differences were found regarding online facilitation and the personality traits openness, conscientiousness, and agreeableness, with degree-seeking students rating higher than short-term exchange students. By contrast, short-term exchange students rated significantly higher for neuroticism than degree-seeking students.

To determine the role of personality traits in predicting study abroad difficulties, several hierarchical multiple regression analyses were conducted. The background demographic variables age, gender, duration of stay, and status were used as control variables in the analyses (stage 1). Similarly, internet use subscales (online benefits, online habits, and online facilitation) were held constant (stage 2). An overall summary of the hierarchical multiple regression analyses is shown in Table 9. The findings show that when controlling for the background demographics and internet use subscales, the personality trait neuroticism consistently showed a positive association with study abroad difficulties. By contrast, some of the other personality traits (except openness) were found to have significant negative effects on study abroad difficulties, although these results were not as consistent.

Table 9. Summary of hierarchical multiple regressions results.

\begin{tabular}{|c|c|c|c|c|c|c|c|c|}
\hline Stage & Variables & Overall & Academic & Leisure & Viewpoints & Daily & Responsive & Suppressive \\
\hline \multirow{4}{*}{1} & Age & $\sqrt{ }(+)$ & & $\sqrt{ }(+)$ & $\sqrt{ }(+)$ & $\sqrt{ }(+)$ & & $\sqrt{ }(+)$ \\
\hline & Gender & & & & & & & \\
\hline & Duration of stay & $\sqrt{ }(-)$ & & $\sqrt{ }(-)$ & $\sqrt{ }(-)$ & $\sqrt{ }(-)$ & & \\
\hline & Status & $\sqrt{ }(+)$ & $\mathcal{J}(+)$ & & & $\sqrt{ }(+)$ & & \\
\hline \multirow{3}{*}{2} & Online benefits & & & & $\sqrt{ }(-)$ & & & \\
\hline & Online habits & & & & & & $\sqrt{ }(-)$ & $\sqrt{ }(-)$ \\
\hline & $\begin{array}{c}\text { Online } \\
\text { facilitation }\end{array}$ & $\sqrt{ }(-)$ & $\sqrt{ }(-)$ & $\sqrt{ }(-)$ & & $\sqrt{ }(-)$ & $\sqrt{ }(-)$ & $\sqrt{ }(-)$ \\
\hline \multirow{5}{*}{3} & Openness & & & & & & & \\
\hline & Conscientiousness & & $\sqrt{ }(-)$ & & $\sqrt{ }(-)$ & $\sqrt{ }(+)$ & & \\
\hline & Extraversion & & $\sqrt{ }(-)$ & & & & $\mathcal{S}(-)$ & \\
\hline & Agreeableness & $\sqrt{ }(-)$ & & $\sqrt{ }(-)$ & & $\sqrt{ }(-)$ & $\sqrt{ }(-)$ & $\sqrt{ }(-)$ \\
\hline & Neuroticism & $\mathcal{S}(+)$ & $\sqrt{ }(+)$ & $\sqrt{ }(+)$ & $\sqrt{ }(+)$ & $\sqrt{ }(+)$ & $\sqrt{ }(+)$ & $\sqrt{ }(+)$ \\
\hline
\end{tabular}

Notes. $\checkmark$ = significant predictors. $(-)$ negative or (+) positive association with the dependent variable.

Widiger [67] explained neuroticism as the tendency of individuals to experience negative emotions. He also reported that individuals who rated high for neuroticism are more likely to experience anxiety and depression. In study abroad students, neuroticism is commonly correlated with the stress associated with experiencing something new and unfamiliar [93,94]. In a longitudinal study, Jeronimus et al. [104] found that neuroticism consistently predicted negative experiences. However, both Andrews et al. [105] and Niehoff et al. [42] suggested that study abroad experiences help reduce the levels of neuroticism. The findings of the current study suggest that neuroticism is closely related to study abroad difficulties. In other words, students who rated high for neuroticism tended to experience greater difficulty while studying abroad.

When examining the moderating effects of personality traits on the relationship between internet use and study abroad difficulties, background demographics were treated as control variables. The findings reveal that openness, extraversion, agreeableness, and neuroticism all showed significant interactions with internet use, suggesting a moderating effect on the relationship between internet use and study abroad difficulties. In other words, although neuroticism by itself is positively associated with study abroad difficulties, when the relationship between internet use and study abroad difficulties was considered, openness, extraversion, agreeableness, and neuroticism all exhibited a moderating role. 
More specifically, openness, extraversion, and agreeableness were found to strengthen the negative relationship between internet use and study abroad difficulties, while neuroticism was found to strengthen the positive relationship between internet use and study abroad difficulties. These findings are unique and contribute to a better understanding of how individual personality traits affect study abroad experiences.

Lastly, to further understand the effects of extreme personalities—-high personality traits $(+2 \mathrm{SD})$ and low personality traits $(-2 \mathrm{SD})$ - simple slopes difference tests were performed. The findings show significant differences between all the high personality traits and their lower counterparts. Further analyses of the results revealed particularly interesting findings. While the moderating effect of conscientiousness was not statistically significant in the previous analysis, simple slopes comparisons showed that when the sample was separated into high $(+2 \mathrm{SD}, n=48)$, mean $(n=1781)$, and low $(-2 \mathrm{SD}, n=41)$ conscientiousness, the moderating effects of both high and mean conscientiousness were, in fact, significant (see Table 5, simple slopes models). Furthermore, for the personality traits openness, extraversion, and agreeableness, the moderating effects of low ( $-2 \mathrm{SD})$ ratings were also not significant. Figures 2, 4 and 5 show that low (blue broken lines) ratings for personality traits exhibit small to very small slopes (almost a straight line for agreeableness), signifying that study abroad difficulties are almost not affected at all by internet use. Likewise, the moderating effect of high (+2 SD) neuroticism was not significant. Importantly, Figure 6 shows that the slope for high neuroticism (red line) is almost a straight line, denoting that study abroad difficulties are not affected at all by internet use.

It should be noted that this study is not without limitations. The data analysis excludes some information regarding students' personal, situational, and contextual characteristics that may also influence the difficulties they may face when studying abroad, which is currently beyond the scope of the study. For instance, these include students' country of origin, Mandarin Chinese language proficiency, discipline of study, housing, host institutions' ranking, governance, and location. Future studies are encouraged to examine these variables either as a predictor or as an antecedent of study abroad difficulties.

\section{Conclusions}

In sum, this study reveals several pertinent findings. First, descriptive, correlational, and group (independent samples $t$-tests) analyses showed that background demographic variables seemed to exert some influence on internet use, study abroad difficulties, and personality traits. Second, background demographic variables and internet use were controlled to determine for the effects of personality traits on study abroad difficulties. The findings show that neuroticism consistently exhibited positive associations with study abroad difficulties. Third, to determine the moderating effects of personality traits on the relationship between internet use and study abroad difficulties, background demographic variables were controlled. The findings reveal that all personality traits except conscientiousness (openness, extraversion, agreeableness, and neuroticism) showed significant interactions with internet use, which implies that these personality traits do moderate the relationship between internet use and study abroad difficulties. Lastly, to further understand the effects of extreme personalities, a comparison between high (+2 SD) and low $(-2 \mathrm{SD})$ personality traits was performed using simple slope differences while controlling for the background demographic variables. All variables were standardized and centered prior to the analyses. The findings show significant differences between all the high personality traits and their lower counterparts, which suggests that while some personality traits moderate the relationship between internet use and study abroad difficulties, the levels of the personality traits also matter.

Apart from the fact that international student offices can use personality scales to identify at-risk students, some practical implications can also be drawn. For instance, international student offices can organize self-discovery workshops, so students can also be made aware of their personalities. Furthermore, interaction between the local Taiwanese 
students can be encouraged with the help of study groups for academic assistance and sightseeing tours for tourism purposes. In addition, with the help of international student offices, study abroad students can also act as cultural ambassadors and help promote their home country. Ultimately, increased self-awareness, self-understanding, and interaction with the local community should help ease the students' acculturation process and therefore lead to a more satisfying study abroad experience.

Funding: Data collection was funded by the Taiwan Ministry of Science and Technology under grant number NSC 102-2410-H-030-086-SS2. APC was funded in part by Fu Jen Catholic University, Taiwan.

Institutional Review Board Statement: The data collection procedure was accomplished in accordance with the guidelines of the Declaration of Helsinki for the protection of human research subjects. In addition, the protocol of the study was reviewed and approved by the committee members of the $\mathrm{Fu}$ Jen Catholic University Institutional Review Board under Case Number C102015.

Informed Consent Statement: Informed consent was obtained from all subjects involved in the study.

Data Availability Statement: Data for the current study is available at https:/ / doi.org/10.6084/m9 .figshare.14812068.v1 (accessed on 20 June 2021).

Acknowledgments: Some parts of an earlier stage of the data analysis was presented in a poster session during the 13th Biennial Asian Association of Social Psychology Conference held at Taipei, Taiwan on 11 to 13 July 2019.

Conflicts of Interest: The author declare no conflict of interest.

\section{References}

1. Di Giovine, M.A.; de Uriarte, J.B. Questioning the future of study abroad in a post-COVID-19 world. In Study Abroad and the Quest for an Anti-Tourism Experience; de Uriarte, J.B., Di Giovine, M.A., Eds.; Lexington Books: Lanham, MD, USA, 2021 ; pp. 325-345.

2. Pan, S. COVID-19 and the neo-liberal paradigm in higher education: Changing landscape. Asian Educ. Dev. Stud. 2020, 10, 322-335. [CrossRef]

3. Edex Live. Over 91 Per Cent Indian Students Want to Study Abroad Post-COVID: ISchoolConnect Survey. Available online: https://www.edexlive.com/news/2020/sep/23/over-91-per-cent-indian-students-want-to-study-abroad-postcovidischoolconnect-survey-14781.html (accessed on 3 May 2021).

4. Pang, C. The Impact of COVID-19 on Study Abroad: Oct 2020 Survey Results. Available online: https:/ / institutions.educations. com/insights/the-impact-of-covid-19-on-study-abroad-oct-2020-survey-results (accessed on 3 May 2021).

5. Dietrich, A.J. Charting a Path Forward for Education Abroad Research. Front. Interdiscip. J. Study Abroad 2020, 32, 1-11. [CrossRef]

6. Takayaa, K. Needs Assessment for Exchange Students in Taiwan. J. Int. Comp. Educ. 2016, 5, 33-46. [CrossRef]

7. Nguyen, T.N.-A.; Cao, T.Q.; Pham, H.-H. Impact of the new southbound policies in international students on Taiwan: An exploratory study from vietnamese oversea students. In Higher Education in Market-Oriented Socialist Vietnam; Phan, L.H., Doan, B.N., Eds.; Palgrave Macmillan: Cham, Switzerland, 2020; pp. 227-249.

8. Chan, S.-J. Recruiting students from China: Taiwan's policies and the dilemmas it faces. Chin. Educ. Soc. 2014, 47, 65-82. [CrossRef]

9. Ministry of Education. International Students Come Together to Taiwan, Where the One-Hundred Thirty Thousand Overseas Student Mark Has Been Passed. Available online: https:/ / english.moe.gov.tw/cp-117-25416-d8868-1.html (accessed on 14 July 2021).

10. Ministry of Education. Statistics on the Number of Foreign Students in Colleges and Universities. Available online: https: / / data.gov.tw/ dataset/6289 (accessed on 14 July 2021).

11. Collier, S. 8 of the Best Places to Study Abroad in Asia. Available online: https://www.topuniversities.com/blog/8-best-placesstudy-abroad-asia (accessed on 16 July 2021).

12. Quacquarelli Symonds. Destination Guides: Study in Taiwan. Available online: https://www.topuniversities.com/where-tostudy/asia/taiwan/guide (accessed on 16 July 2021).

13. McKeown, J.S. The First Time Effect: The Impact of Study Abroad on College Student Intellectual Development; State University of New York Press: Albany, NY, USA, 2009.

14. Ryan, M.E.; Twibell, R.S. Concerns, values, stress, coping, health and educational outcomes of college students who studied abroad. Int. J. Intercult. Relat. 2000, 24, 409-435. [CrossRef]

15. Nguyen, M.-H.; Serik, M.; Vuong, T.-T.; Ho, M.-T. Internationalization and Its Discontents: Help-Seeking Behaviors of Students in a Multicultural Environment Regarding Acculturative Stress and Depression. Sustainability 2019, 11, 1865. [CrossRef] 
16. Machul, M.; Bieniak, M.; Chałdaś-Majdańska, J.; Bąk, J.; Chrzan-Rodak, A.; Mazurek, P.; Pawłowski, P.; Makuch-Kuśmierz, D.; Obuchowska, A.; Bartoszek, A.; et al. Lifestyle Practices, Satisfaction with Life and the Level of Perceived Stress of Polish and Foreign Medical Students Studying in Poland. Int. J. Environ. Res. Public Health 2020, 17, 4445. [CrossRef] [PubMed]

17. Kabir, R.; Isha, S.; Chowdhury, M.; Siddika, N.; Jahan, S.; Saha, A.; Nath, S.; Jahan, M.; Sivasubramanian, M.; Mahmud, I.; et al. Depression among the Non-Native International Undergraduate Students Studying Dentistry in Bangladesh. Int. J. Environ. Res. Public Health 2021, 18, 5802. [CrossRef]

18. Huang, L.; Kern, M.L.; Oades, L.G. Strengthening University Student Wellbeing: Language and Perceptions of Chinese International Students. Int. J. Environ. Res. Public Health 2020, 17, 5538. [CrossRef]

19. Ward, C.A.; Bochner, S.; Furnham, A. The Psychology of Culture Shock, 2nd ed.; Routledge: London, UK, 2001.

20. Dykhouse, E.C.; Bikos, L.H. Re-entry friction: The curious effects of cultural dislocation on outcomes for global service learning returnees. Int. J. Intercult. Relat. 2019, 72, 96-108. [CrossRef]

21. Wielkiewicz, R.M.; Turkowski, L.W. Reentry Issues upon Returning From Study Abroad Programs. J. Coll. Stud. Dev. 2010, 51, 649-664. [CrossRef]

22. Zhang, H.; Gibson, H. Long-Term Impact of Study Abroad on Sustainability-Related Attitudes and Behaviors. Sustainability 2021, 13, 1953. [CrossRef]

23. Mazzarol, T. Critical success factors for international education marketing. Int. J. Educ. Manag. 1998, 12, 163-175. [CrossRef]

24. Rhodes, G.M. The Internet and World Wide Web: Uses for Study Abroad. Front. Interdiscip. J. Study Abroad 1995, 1, 108-112. [CrossRef]

25. Coleman, J.A.; Chafer, T. Study abroad and the internet: Physical and virtual context in an era of expanding telecommunications. Front. Interdiscip. J. Study Abroad 2010, 19, 151-168. [CrossRef]

26. Woolley, S. Constantly connected: The impact of social media and the advancement in technology on the study abroad experience. Elon J. Undergrad. Res. Commun. 2013, 4, 36-46.

27. Lin, M.-C.; Ching, G.S. The role of internet in study abroad related stress in Taiwan. In Society for Information Technology E Teacher Education International Conference; Rutledge, D., Slykhuis, D., Eds.; Association for the Advancement of Computing in Education: Las Vegas, NV, USA, 2015; pp. 1207-1211.

28. Mikal, J.P.; Grace, K. Against abstinence-only education abroad: Viewing internet use during study abroad as a possible experience enhancement. J. Stud. Int. Educ. 2011, 16, 287-306. [CrossRef]

29. Mikal, J.P.; Yang, J.; Lewis, A. Surfing USA: How internet use prior to and during study abroad affects Chinese students' stress, integration, and cultural learning while in the United States. J. Stud. Int. Educ. 2014, 19, 203-224. [CrossRef]

30. Sauer, L.; Ellis, R. The Social Lives of Adolescent Study Abroad Learners and Their L2 Development. Mod. Lang. J. 2019, 103, 739-762. [CrossRef]

31. Basow, S.A.; Gaugler, T. Predicting adjustment of U.S. college students studying abroad: Beyond the multicultural personality. Int. J. Intercult. Relat. 2017, 56, 39-51. [CrossRef]

32. Pedersen, E.R.; Neighbors, C.; Larimer, M.E.; Lee, C.M. Measuring Sojourner Adjustment among American students studying abroad. Int. J. Intercult. Relat. 2011, 35, 881-889. [CrossRef]

33. Duranczyk, I.; Pishcherskaia, E. Bridging Countries and Cultures through Accessible Global Collaborations. Educ. Sci. 2018, 8, 199. [CrossRef]

34. Bakalis, S.; Joiner, T.A. Participation in tertiary study abroad programs: The role of personality. Int. J. Educ. Manag. 2004, 18, 286-291. [CrossRef]

35. Li, M.; Olson, J.E.; Frieze, I.H. Students' Study Abroad Plans: The Influence of Motivational and Personality Factors. Front. Interdiscip. J. Study Abroad 2013, 23, 73-89. [CrossRef]

36. Leong, C.-H. Predictive validity of the Multicultural Personality Questionnaire: A longitudinal study on the socio-psychological adaptation of Asian undergraduates who took part in a study-abroad program. Int. J. Intercult. Relat. 2007, 31, 545-559. [CrossRef]

37. Ramirez, E.R. Impact on Intercultural Competence When Studying Abroad and the Moderating Role of Personality. J. Teach. Int. Bus. 2016, 27, 88-105. [CrossRef]

38. Wang, W.-L.; Ching, G.S. The role of personality and intercultural effectiveness towards study abroad academic and social activities. Int. J. Res. Stud. Psychol. 2015, 4, 13-27. [CrossRef]

39. Goel, L.; De Jong, P.; Schnusenberg, O. Toward a Comprehensive Framework of Study Abroad Intentions and Behaviors. J. Teach. Int. Bus. 2010, 21, 248-265. [CrossRef]

40. Digman, J.M. Personality Structure: Emergence of the Five-Factor Model. Annu. Rev. Psychol. 1990, 41, 417-440. [CrossRef]

41. Goldberg, L.R. The structure of phenotypic personality traits. Am. Psychol. 1993, 48, 26-34. [CrossRef]

42. Niehoff, E.; Petersdotter, L.; Freund, P.A. International sojourn experience and personality development: Selection and socialization effects of studying abroad and the Big Five. Pers. Individ. Differ. 2017, 112, 55-61. [CrossRef]

43. Chmielewski, M.S.; Morgan, T.A. Five-factor model of personality. In Encyclopedia of Behavioral Medicine; Gellman, M.D., Turner, J.R., Eds.; Springer: New York, NY, USA, 2013.

44. Yakunina, E.S.; Weigold, I.K.; Weigold, A.; Hercegovac, S.; Elsayed, N. The multicultural personality: Does it predict international students' openness to diversity and adjustment? Int. J. Intercult. Relat. 2012, 36, 533-540. [CrossRef]

45. Ramírez-Correa, P.; Grandón, E.E.; Alfaro-Pérez, J.; Painén-Aravena, G. Personality Types as Moderators of the Acceptance of Information Technologies in Organizations: A Multi-Group Analysis in PLS-SEM. Sustainability 2019, 11, 3987. [CrossRef] 
46. Stodt, B.; Brand, M.; Sindermann, C.; Wegmann, E.; Li, M.; Zhou, M.; Sha, P.; Montag, C. Investigating the Effect of Personality, Internet Literacy, and Use Expectancies in Internet-Use Disorder: A Comparative Study between China and Germany. Int. J. Environ. Res. Public Health 2018, 15, 579. [CrossRef]

47. Yu, T.-K.; Lee, N.-H.; Chao, C.-M. The Moderating Effects of Young Adults' Personality Traits on Social Media Immersion. Front. Psychol. 2020, 11, 2931. [CrossRef]

48. Durak, E.S.; Durak, M. Which personality traits are associated with cognitions related to problematic Internet use? Asian J. Soc. Psychol. 2014, 17, 206-218. [CrossRef]

49. Muscanell, N.L.; Guadagno, R.E. Make new friends or keep the old: Gender and personality differences in social networking use. Comput. Hum. Behav. 2012, 28, 107-112. [CrossRef]

50. Servidio, R. A Discriminant Analysis to Predict the Impact of Personality Traits, Self-esteem, and Time Spent Online on Different Levels of Internet Addiction Risk among University Students. Stud. Psychol. 2019, 61, 56-70. [CrossRef]

51. Ruggieri, R.A.; Iervolino, A.; Mossi, P.; Santoro, E.; Boccia, G. Instability of Personality Traits of Teachers in Risk Conditions due to Work-Related Stress. Behav. Sci. 2020, 10, 91. [CrossRef]

52. Babbie, E.R. The Practice of Social Research, 12th ed.; Wadsworth Cengage: Belmont, CA, USA, 2010.

53. Muijs, D. Doing Quantitative Research in Education with SPSS, 2nd ed.; SAGE Publications: London, UK, 2010.

54. Stewart, D.W.; Shamdasani, P.N. Focus Groups: Theory and Practice, 3rd ed.; Sage: Thousand Oaks, CA, USA, 2014.

55. Glaziou, P. Sampsize. Available online: http://sampsize.sourceforge.net/iface/ (accessed on 16 July 2021).

56. Madley-Dowd, P.; Hughes, R.; Tilling, K.; Heron, J. The proportion of missing data should not be used to guide decisions on multiple imputation. J. Clin. Epidemiol. 2019, 110, 63-73. [CrossRef]

57. Graham, J.W. Missing Data Analysis: Making It Work in the Real World. Annu. Rev. Psychol. 2009, 60, 549-576. [CrossRef]

58. Cronbach, L.J. Coefficient alpha and the internal structure of tests. Psychometrika 1951, 16, 297-334. [CrossRef]

59. Cohen, L.; Manion, L.; Morrison, K. Research Method in Education, 6th ed.; Routledge: London, UK, 2007.

60. John, O.P.; Srivastava, S. The big five trait taxonomy: History, measurement, and theoretical perspectives. In Handbook of Personality: Theory and Research, 2nd ed.; Pervin, L.A., John, O.P., Eds.; Guilford Press: New York, NY, USA, 1999 ; pp. $102-138$.

61. Likert, R. A Technique for the Measurement of Attitudes; Columbia University Press: New York, NY, USA, 1932.

62. DeYoung, C.G. Openness/intellect: A dimension of personality reflecting cognitive exploration. In APA Handbook of Personality and Social Psychology: Personality Processes and Individual Differences; Mikulincer, M., Shaver, P.R., Cooper, M.L., Larsen, R.J., Eds.; American Psychological Association: Washington, DC, USA, 2015; Volume 4, pp. 369-399.

63. Roberts, B.W.; Jackson, J.J.; Fayard, J.V.; Edmonds, G.; Meints, J. Conscientiousness. In Handbook of Individual Differences in Social Behavior; Leary, M.R., Hoyle, R.H., Eds.; Guilford Press: New York, NY, USA, 2009; pp. 369-381.

64. Watson, D.; Clark, L.A. Extraversion and its positive emotional core. In Handbook of Personality Psychology; Hogan, R., Johnson, J., Briggs, S., Eds.; Academic Press: Cambridge, MA, USA, 1997; pp. 767-793.

65. Wilt, J.; Revelle, W. Extraversion. In Handbook of Individual Differences in Social Behavior; Leary, M.R., Hoyle, R.H., Eds.; Guilford Press: New York, NY, USA, 2009; pp. 27-45.

66. Graziano, W.G.; Tobin, R.M. Agreeableness. In Handbook of Individual Differences in Social Behavior; Leary, M.R., Hoyle, R.H., Eds.; Guilford Press: New York, NY, USA, 2009; pp. 46-61.

67. Widiger, T.A. Neuroticism. In Handbook of Individual Differences in Social Behavior; Leary, M.R., Hoyle, R.H., Eds.; Guilford Press: New York, NY, USA, 2009; pp. 129-146.

68. Anderson, J.C.; Gerbing, D.W. Structural equation modeling in practice: A review and recommended two-step approach. Psychol. Bull. 1988, 103, 411-423. [CrossRef]

69. Ho, R. Handbook of Univariate and Multivariate Data Analysis and Interpretation with IBM SPSS; Taylor and Francis: New York, NY, USA, 2006.

70. Hu, L.T.; Bentler, P.M. Cutoff criteria for fit indexes in covariance structure analysis: Conventional criteria versus new alternatives. Struct. Equ. Model. Multidiscip. J. 1999, 6, 1-55. [CrossRef]

71. Byrne, B.M. Structural equation modeling with AMOS. In Basic Concepts, Applications, and Programming, 2nd ed.; Taylor \& Francis: New York, NY, USA, 2010.

72. Cho, G.; Hwang, H.; Sarstedt, M.; Ringle, C.M. Cutoff criteria for overall model fit indexes in generalized structured component analysis. J. Mark. Anal. 2020, 8, 189-202. [CrossRef]

73. Marsh, H.; Hocevar, D. Application of confirmatory factor analysis to the study of self-concept: First- and higher order factor models and their invariance across groups. Psychol. Bull. 1985, 97, 562-582. [CrossRef]

74. Ching, G.S.; Lien, W.-C.; Chao, P.-C. Developing a scale to measure the situational changes in short-term study abroad programs. Int. J. Res. Stud. Educ. 2014, 3, 53-71. [CrossRef]

75. Soper, D.S. Interaction! Available online: https://www.danielsoper.com/interaction/default.aspx (accessed on 5 January 2021).

76. Fornell, C.; Larcker, D.F. Evaluating Structural Equation Models with Unobservable Variables and Measurement Error. J. Mark. Res. 1981, 18, 39-50. [CrossRef]

77. Cohen, J. Statistical Power Analysis for the Behavioral Sciences, 2nd ed.; Lawrence Erlbaum Associates: Hillsdale, NJ, USA, 1988; p. 567.

78. Dawson, J.F.; Richter, A.W. Probing three-way interactions in moderated multiple regression: Development and application of a slope difference test. J. Appl. Psychol. 2006, 91, 917-926. [CrossRef] 
79. Altman, D.G.; Bland, J.M. Standard deviations and standard errors. BMJ 2005, 331, 903. [CrossRef]

80. Dalal, D.K.; Zickar, M.J. Some Common Myths About Centering Predictor Variables in Moderated Multiple Regression and Polynomial Regression. Organ. Res. Methods 2011, 15, 339-362. [CrossRef]

81. Laubscher, N.F. Normalizing the noncentral t and F distributions. Ann. Math. Stat. 1960, 31, 1105-1112. [CrossRef]

82. Robinson, C.D.; Tomek, S.; Schumacker, R.E. Tests of moderation effects: Difference in simple slopes versus the interaction term. Mult. Linear Regres. Viewp. 2013, 39, 16-24.

83. Webb, M.W. Cross-Cultural Awareness: A Framework for Interaction. Pers. Guid. J. 1983, 61, 498-500. [CrossRef]

84. Mapp, S.C.; McFarland, P.; Newell, E.A. The Effect of a Short-Term Study Abroad Class on Students' Cross-Cultural Awareness . J. Bac. Soc. Work. 2007, 13, 39-51. [CrossRef]

85. Hamad, R.; Lee, C.M. An Assessment of How Length of Study-Abroad Programs Influences Cross-Cultural Adaptation. J. Hum. Behav. Soc. Environ. 2013, 23, 661-674. [CrossRef]

86. Onosu, G. The Impact of Cultural Immersion Experience on Identity Transformation Process. Int. J. Environ. Res. Public Health 2021, 18, 2680. [CrossRef]

87. Su, Y. Promoting cross-cultural awareness and understanding: Incorporating ethnographic interviews in college EFL classes in Taiwan. Educ. Stud. 2008, 34, 377-398. [CrossRef]

88. Langley, C.S.; Breese, J.R. Interacting sojourners: A study of students studying abroad. Soc. Sci. J. 2005, 42, 313-321. [CrossRef]

89. Quezada, R.L. Beyond educational tourism: Lessons learned while student teaching abroad. Int. Educ. J. 2004, 5, 458-465.

90. Breen, M. Privileged migration: American undergraduates, study abroad, academic tourism. Crit. Arts 2012, 26, 82-102. [CrossRef]

91. Lee, C.-F. Exploring Motivations for Studying Abroad: A Case Study of Taiwan. Tour. Anal. 2017, 22, 523-536. [CrossRef]

92. Mikal, J. When Social Support Fits into your Luggage: Online Support Seeking and its Effects on the Traditional Study Abroad Experience. Front. Interdiscip. J. Study Abroad 2011, 21, 17-40. [CrossRef]

93. Kong, D.T. The Role of Mindfulness and Neuroticism in Predicting Acculturative Anxiety Forecasting Error. Mindfulness 2015, 6, 1387-1400. [CrossRef]

94. Kong, D.T. Sojourners' Ineffective Sociocultural Adaptation: Paranoia as a Joint Function of Distrust toward Host Nationals and Neuroticism. Curr. Psychol. 2016, 36, 540-548. [CrossRef]

95. Alcón-Soler, E. Pragmatic learning and study abroad: Effects of instruction and length of stay. System 2015, 48, 62-74. [CrossRef]

96. Briggs, J.G. Out-of-class language contact and vocabulary gain in a study abroad context. System 2015, 53, 129-140. [CrossRef]

97. Kim, H.J.; Okazaki, S. Navigating the cultural transition alone: Psychosocial adjustment of Korean early study abroad students. Cult. Divers. Ethn. Minor. Psychol. 2014, 20, 244-253. [CrossRef] [PubMed]

98. Niehaves, B.; Plattfaut, R. Internet adoption by the elderly: Employing IS technology acceptance theories for understanding the age-related digital divide. Eur. J. Inf. Syst. 2014, 23, 708-726. [CrossRef]

99. Chung, J.E.; Park, N.; Wang, H.; Fulk, J.; McLaughlin, M. Age differences in perceptions of online community participation among non-users: An extension of the Technology Acceptance Model. Comput. Hum. Behav. 2010, 26, 1674-1684. [CrossRef]

100. Aleem, S. Emotional stability among college youth. J. Indian Acad. Appl. Psychol. 2005, 31, 100-102.

101. Hills, P.; Argyle, M. Emotional stability as a major dimension of happiness. Pers. Individ. Differ. 2001, 31, 1357-1364. [CrossRef]

102. Hashim, I.H.; Zhiliang, Y. Cultural and gender differences in perceiving stressors: A cross-cultural investigation of African and Western students in Chinese colleges. Stress Health 2003, 19, 217-225. [CrossRef]

103. Yuerong, C.; Susan, L.R.; Samantha, M.; Joni, S.; Anthony, T.S.; Ching, Y.; Renes, S.L.; McMurrow, S.; Simpson, J.; Strange, A.T. Challenges facing Chinese International students studying in the United States. Educ. Res. Rev. 2017, 12, 473-482. [CrossRef]

104. Jeronimus, B.F.; Riese, H.; Sanderman, R.; Ormel, J. Mutual reinforcement between neuroticism and life experiences: A five-wave, 16-year study to test reciprocal causation. J. Pers. Soc. Psychol. 2014, 107, 751-764. [CrossRef] [PubMed]

105. Andrews, G.; Page, A.; Neilson, M.D. Sending your teenagers away: Controlled stress decreases neurotic vulnerability. Arch. Gen. Psychiatry 1993, 50, 585-589. [CrossRef] [PubMed] 